\title{
PHASE PORTRAITS OF ABEL QUADRATIC DIFFERENTIAL SYSTEMS OF SECOND KIND WITH SYMMETRIES
}

\author{
ANTONI FERRAGUT, JOHANNA D. GARCÍA-SALDAÑA, CLAUDIA VALLS
}

\begin{abstract}
We provide normal forms and the global phase portraits on the Poincaré disk of the Abel quadratic differential equations of the second kind having a symmetry with respect to an axis or to the origin. Moreover, we also provide the bifurcation diagrams for these global phase portraits.
\end{abstract}

\section{Introduction AND STATEMENTS OF THE MAIN RESUlts}

Abel differential equations of the second kind are named in honor of Niels Henrik Abel because by a direct substitution they are related with the Abel differential equations of the first kind which were obtained by him in his studies of the theory of elliptic functions (see [11]) and after that, he made a crucial research on them. Abel differential equations of the second kind have various applications as they appear to reduce the order of many higher order nonlinear problems. They are also frequently found in the modeling of real problems such as big picture modeling in oceanic circulation (see [1] and the references therein).

An Abel differential equation of second kind has the form

$$
y \frac{d y}{d x}=A(x) y^{2}+B(x) y+C(x),
$$

with $A(x), B(x), C(x) \in \mathbb{R}(x, y)$. This differential equation can be written equivalently as the polynomial differential system

$$
\dot{x}=d(x) y, \quad \dot{y}=a(x) y^{2}+b(x) y+c(x),
$$

where $a(x), b(x), c(x)$ and $d(x)$ are polynomials such that $A(x)=a(x) / d(x), B(x)=$ $b(x) / d(x)$ and $C(x)=c(x) / d(x)$. In this paper we are interested in studying the Abel quadratic polynomial differential systems, i.e. the differential systems (2) of degree two:

$$
\dot{x}=\left(d_{0}+d_{1} x\right) y, \quad \dot{y}=a_{0} y^{2}+\left(b_{0}+b_{1} x\right) y+c_{0}+c_{1} x+c_{2} x^{2} .
$$

All the parameters in (3) are real. We assume that $\dot{x}$ and $\dot{y}$ do not have a common factor; in particular, we assume that $c_{0}^{2}+c_{1}^{2}+c_{2}^{2} \neq 0$. Moreover, we take $a_{0} \neq 0$, otherwise this is not the Abel equation of second kind. We also assume that $b_{1}^{2}+c_{1}^{2}+c_{2}^{2}+d_{1}^{2} \neq 0$, otherwise the system does not depend on $x$ and hence it is not of our interest.

In this work we study the global phase portraits of the Abel differential systems of second kind of degree two given by system (3) with $d_{1} \neq 0$. The case $d_{1}=0$ is completely studied in [7]. Since this is a huge challenge, we restrict our study to the differential systems (3) having a symmetry with respect to an axis or with respect to the origin, see (4) below. We shall provide all the possible global phase portraits for these families. For that purpose, we shall use the well-known Poincare compactification of polynomial vector fields, see section 2.3. Before stating our main theorem, we split the family (3) into five different families.

Proposition 1. System (3) with $d_{1} \neq 0$ leads, after an affine change of the variables and a scaling of the time, to the differential system $\dot{x}=x y, \dot{y}=Q(x, y)$, where $Q(x, y)$ is one of the following five quadratic polynomials:

(K1) $Q(x, y)=S\left(x-r_{1}\right)\left(x-r_{2}\right)+\left(B_{0}+B_{1} x\right) y+A_{0} y^{2}$;

2010 Mathematics Subject Classification. 34A34, 34C20, 34C23, 34C05.

Key words and phrases. Abel differential equation of second kind, quadratic differential system, phase portrait, Poincaré disk. 
(K2) $Q(x, y)=S(x-r)^{2}+\left(B_{0}+B_{1} x\right) y+A_{0} y^{2}$

(K3) $Q(x, y)=S\left((x-a)^{2}+b^{2}\right)+\left(B_{0}+B_{1} x\right) y+A_{0} y^{2}$;

(K4) $Q(x, y)=x-T+\left(B_{0}+B_{1} x\right) y+A_{0} y^{2}$;

(K5) $Q(x, y)=S+\left(B_{0}+B_{1} x\right) y+A_{0} y^{2}$;

where $S \in\{-1,1\}, T \in\{-1,0,1\}, a, B_{0}, B_{1}, r, r_{1}, r_{2} \in \mathbb{R}, A_{0} \in \mathbb{R} \backslash\{0\}, r_{1} \neq r_{2}$ and $b>0$.

Next proposition splits the families in Proposition 1 into several subfamilies, considering the following three symmetries, mentioned above:

$$
\left(S_{a}\right) \quad x \mapsto-x ; \quad\left(S_{b}\right) \quad y \mapsto-y ; \quad\left(S_{c}\right) \quad(x, y) \mapsto-(x, y) ;
$$

and the possible change of sign of the independent variable, $t \mapsto-t$.

Proposition 2. After applying the three symmetries $\left(S_{a}\right),\left(S_{b}\right)$ and $\left(S_{c}\right)$, the five families in Proposition 1 can be written, after an affine change of the variables and a scaling of the time, as $\dot{x}=x y, \dot{y}=Q(x, y)$, where $Q(x, y)$ is one of the following thirteen quadratic polynomials:

(K1a) $Q(x, y)=S\left(x^{2}-1\right)+B_{0} y+A_{0} y^{2}, B_{0}>0$;

(K1b) $Q(x, y)=S(x-r)(x-1)+A_{0} y^{2}, r \neq 1$;

(K1c) $Q(x, y)=S\left(x^{2}-1\right)+B_{1} x y+A_{0} y^{2}, B_{1} \geq 0$;

(K2a) $Q(x, y)=S x^{2}+y+A_{0} y^{2}$;

(K2b) $Q(x, y)=S(x-r)^{2}+A_{0} y^{2}, r \in\{0,1\}$;

(K2c) $Q(x, y)=S x^{2}+B_{1} x y+A_{0} y^{2}, B_{1}>0$;

(K3a) $Q(x, y)=S\left(x^{2}+b^{2}\right)+B_{0} y+A_{0} y^{2}, B_{0}>0$;

(K3b) $Q(x, y)=S\left((x-a)^{2}+1\right)+A_{0} y^{2}, a \in \mathbb{R}$;

(K3c) $Q(x, y)=S\left(x^{2}+1\right)+B_{1} x y+A_{0} y^{2}, B_{1}>0$

(K4a) $Q(x, y)=x-T+A_{0} y^{2}, T^{2} \in\{0,1\}$;

(K5a) $Q(x, y)=S+B_{0} y+A_{0} y^{2}, B_{0}>0$;

(K5b) $Q(x, y)=S+A_{0} y^{2}$;

(K5c) $Q(x, y)=S+x y+A_{0} y^{2}$.

In all cases, we have $S \in\{-1,1\}$ and $A_{0} \in \mathbb{R} \backslash\{0\}$.

Propositions 1 and 2 are proved in section 3. The main result in this paper is the following theorem, where the global phase portraits on the Poincare disk of all the families of Proposition 2 are provided. Theorem 3 follows after Theorem 4, in which we relate the global phase portraits of Figure 1 and the families in Proposition 2 and the conditions on the parameters of $Q$ for each case.

We say that two polynomial vector fields on the Poincare disk are topologically equivalent if there exists a homeomorphism from one onto the other which sends orbits to orbits preserving or reversing the direction of the flow.

Theorem 3. The families of system (3) with $d_{1} \neq 0$ appearing in Proposition 2 provide 84 non-topologically equivalent phase portraits. All these phase portraits are shown in Figure 1. The phase portraits provided by each family are:

(K1a) (1)-(12).

(K1b) (10)-(24).

(K1c) (10)-(13), (18), (20), (25)-(32).

(K2a) (33)-(37).

(K2b) (38)-(46).

(K2c) (38)-(40), (43), (44), (47), (48).

(K3a) (33)-(37), (49)-(52).

(K3b) (34), (35), (37), (50), (51).

(K3c) (34), (35), (37), (50), (51), (53)-(58).

(K4a) (59)-(70).

(K5a) (71)-(83).

(K5b) (71), (74)-(76), (82)-(84).

(K5c) (35), (37), (51), (53)-(55), (57), (58). 
Let $\mathbb{R}_{0}^{+}=\{x \in \mathbb{R}: x \geq 0\}$ and $\mathbb{R}^{+}=\{x \in \mathbb{R}: x>0\}$. Theorem 3 follows directly after the next theorem.

Theorem 4. Regarding Theorem 3, the parameter spaces modulo the group action and the corresponding bifurcation diagrams are as follows:

(K1a) For the family (K1a) the parameter space is $\left(S, A_{0}, B_{0}\right) \in\{-1,1\} \times(\mathbb{R} \backslash\{0\}) \times \mathbb{R}^{+}$. Let $C_{1}:=B_{0}^{2}+4 A_{0} S$.

When $S=-1$ the global phase portrait is:

(1) for $A_{0}<0$;

(2) for $0<A_{0} \leq 1$ and $C_{1}>0$;

(3) for $0<A_{0} \leq 1$ and $C_{1}=0$;

(4) for $0<A_{0} \leq 1$ and $C_{1}<0$;

(5) for $A_{0}>1$ and $C_{1}>0$;

(6) for $A_{0}>1$ and $C_{1}=0$;

(7) for $A_{0}>1$ and $C_{1}<0$.

The bifurcation diagram is shown in Figure 2(a).

When $S=1$ the global phase portrait is:

(8) for $A_{0}<0$ and $C_{1}>0$;

(9) for $A_{0}<0$ and $C_{1}=0$;

(10) for $A_{0}<0$ and $C_{1}<0$;

(11) for $0<A_{0}<1$;

(12) for $A_{0} \geq 1$.

The bifurcation diagram is shown in Figure 2(b).

(K1b) For the family (K1b) the parameter space is $\left(S, A_{0}, r\right) \in\{-1,1\} \times(\mathbb{R} \backslash\{0\}) \times(\mathbb{R} \backslash\{1\})$.

When $S=-1$ the global phase portrait is:

(13) for either $A_{0}=1$ and $r<0$, or $0<A_{0}<1$ and $r<0$;

(14) for either $A_{0}<0$ and $0 \leq r<1$, or $A_{0}<0$ and $r>1$;

(15) for either $0<A_{0} \leq 1$ and $0<r<1$, or $0<A_{0} \leq 1$ and $r>1$;

(16) for either $A_{0}>1$ and $0<r<1$, or $A_{0}>1$ and $r>1$;

(17) for $A_{0}>1$ and $r=0$;

(18) for $A_{0}>1$ and $r<0$;

(19) for $0<A_{0} \leq 1$ and $r=0$;

(20) for $A_{0}<0$ and $r<0$.

The bifurcation diagram is shown in Figure 2(c).

When $S=1$ the global phase portrait is:

(10) for $A_{0}<0$ and $r \leq 0$;

(11) for $0<A_{0}<1$ and $r<0$;

(12) for $A_{0} \geq 1$ and $r<0$;

(14) for either $A_{0} \geq 1$ and $0<r<1$ or $A_{0} \geq 1$ and $r>1$;

(21) for either $A_{0}<0$ and $0<r<1$ or $A_{0}<0$ and $r>1$;

(22) for either $0<A_{0}<1$ and $0<r<1$ or $0<A_{0}<1$ and $r>1$;

(23) for $0<A_{0}<1$ and $r=0$;

(24) for $A_{0} \geq 1$ and $r=0$.

The bifurcation diagram is shown in Figure 2(d).

(K1c) For the family (K1c) the parameter space is $\left(S, A_{0}, B_{1}\right) \in\{-1,1\} \times(\mathbb{R} \backslash\{0\}) \times \mathbb{R}_{0}^{+}$. Let $C_{2}:=B_{1}^{2}-4 A_{0} S+4 S$.

When $S=-1$ the global phase portrait is:

(13) for either $0<A_{0}<1$ and $C_{2}<0$ or $0<A_{0} \leq 1$ and $B_{1}=0$;

(18) for $A_{0}>1$ and $B_{1}=0$;

(20) for $A_{0}<0$ and $B_{1}=0$;

(25) for $A_{0}<0$ and $C_{2}<0$;

(26) for $A_{0}<0$ and $C_{2}=0$;

(27) for $A_{0}<0$ and $C_{2}>0$; 
(28) for $0<A_{0}<1$ and $C_{2}>0$;

(29) for $0<A_{0}<1$ and $C_{2}=0$;

(30) for $A_{0}>1$ and $B_{1}>0$;

(31) for $A_{0}=1$ and $B_{1}>0$.

The bifurcation diagram is shown in Figure 2(e).

When $S=1$ the global phase portrait is:

(10) for $A_{0}<0$ and $B_{1} \geq 0$;

(11) for either $0<A_{0}<1$ and $B_{1} \geq 0$ or $A_{0}>1$ and $C_{2}>0$;

(12) for either $A_{0} \geq 1$ and $B_{1}=0$ or $A_{0}>1$ and $C_{2}<0$;

(32) for $A_{0}=1$ and $B_{1}>0$.

The bifurcation diagram is shown in Figure $2(f)$.

(K2a) For the family (K2a) the parameter space is $\left(S, A_{0}\right) \in\{-1,1\} \times(\mathbb{R} \backslash\{0\})$.

When $S=-1$ the global phase portrait is:

(33) for $A_{0}<0$;

(34) for $0<A_{0} \leq 1$;

(35) for $A_{0}>1$.

When $S=1$ the global phase portrait is:

(33) for $A_{0} \geq 1$;

(36) for $0<A_{0}<1$;

(37) for $A_{0}<0$.

(K2b) For the family (K2b) the parameter space is $\left(S, r, A_{0}\right) \in\{-1,1\} \times\{0,1\} \times(\mathbb{R} \backslash\{0\})$.

When $S=-1$ and $r=0$ the global phase portrait is:

(38) for $A_{0}<0$;

(39) for $0<A_{0} \leq 1$;

(40) for $A_{0}>1$;

When $S=-1$ and $r=1$ the global phase portrait is:

(38) for $A_{0}<0$;

(41) for $0<A_{0} \leq 1$;

(42) for $A_{0}>1$.

When $S=1$ and $r=0$ the global phase portrait is:

(38) for $A_{0} \geq 1$;

(43) for $0<A_{0}<1$;

(44) for $A_{0}<0$.

When $S=1$ and $r=1$ the global phase portrait is:

(38) for $A_{0} \geq 1$;

(45) for $0<A_{0}<1$;

(46) for $A_{0}<0$.

(K2c) For the family (K2c) the parameter space is $\left(S, A_{0}, B_{1}\right) \in\{-1,1\} \times(\mathbb{R} \backslash\{0\}) \times \mathbb{R}^{+}$.

Let $C_{2}:=B_{1}^{2}-4 A_{0} S+4 S$

When $S=-1$ the global phase portrait is:

(38) for $A_{0}<0$ and $C_{2}<0$;

(39) for $0<A_{0}<1$ and $C_{2}<0$;

(40) for either $0<A_{0}<1$ and $C_{2}>0$ or $A_{0}>1$;

(43) for $A_{0}<0$ and $C_{2}>0$;

(47) for $A_{0}<0$ and $C_{2}=0$;

(48) for either $0<A_{0}<1$ and $C_{2}=0$ or $A_{0}=1$.

The bifurcation diagram is shown in Figure $2(\mathrm{~g})$.

When $S=1$ the global global phase portrait is:

(38) for $A_{0}>1$ and $C_{2}<0$;

(43) for either $0<A_{0}<1$ or $A_{0}>1$ and $C_{2}>0$;

(44) for $A_{0}<0$;

(47) for either $A_{0}=1$ or $A_{0}>1$ and $C_{2}=0$;

The bifurcation diagram is shown in Figure $2(h)$. 
(K3a) For the family (K3a) the parameter space is $\left(S, A_{0}, B_{0}\right) \in\{-1,1\} \times(\mathbb{R} \backslash\{0\}) \times \mathbb{R}^{+}$. Let $C_{3}:=B_{0}^{2}-4 A_{0} S$

When $S=-1$ the global phase portrait is:

(33) for $A_{0}<0$ and $C_{3}>0$;

(34) for $0<A_{0} \leq 1$;

(35) for $A_{0}>1$;

(49) for $A_{0}<0$ and $C_{3}=0$;

(50) for $A_{0}<0$ and $C_{3}<0$.

The bifurcation diagram is shown in Figure 2(i).

When $S=1$ the global global phase portrait is:

(33) for $A_{0} \geq 1$ and $C_{3}>0$;

(36) for $0<A_{0}<1$ and $C_{3}>0$;

(37) for $A_{0}<0$;

(49) for $A_{0} \geq 1$ and $C_{3}=0$;

(50) for $A_{0} \geq 1$ and $C_{3}<0$;

(51) for $0<A_{0}<1$ and $C_{3}<0$;

(52) for $0<A_{0}<1$ and $C_{3}=0$.

The bifurcation diagram is shown in Figure $2(j)$.

(K3b) For the family (K3b) the parameter space is $\left(S, A_{0}\right) \in\{-1,1\} \times(\mathbb{R} \backslash\{0\})$.

When $S=-1$ the global phase portrait is:

(34) for $0<A_{0} \leq 1$;

(35) for $A_{0}>1$;

(50) for $A_{0}<0$.

When $S=1$ the global phase portrait is:

(37) for $A_{0}<0$;

(50) for $A_{0} \geq 1$;

(51) for $0<A_{0}<1$.

(K3c) For the family (K3c) the parameter space is $\left(S, A_{0}, B_{1}\right) \in\{-1,1\} \times(\mathbb{R} \backslash\{0\}) \times \mathbb{R}^{+}$. Let $C_{2}:=B_{1}^{2}-4 A_{0} S+4 S$

When $S=-1$ the global phase portrait is:

(34) for $0<A_{0}<1$ and $C_{2}<0$;

(35) for $A_{0}>1$;

(50) for $A_{0}<0$ and $C_{2}<0$;

(53) for either $0<A_{0}<1$ and $C_{2}=0$ or $A_{0}=1$;

(54) for $0<A_{0}<1$ and $C_{2}>0$;

(55) for $A_{0}<0$ and $C_{2}>0$;

(56) for $A_{0}<0$ and $C_{2}=0$.

The bifurcation diagram is shown in Figure 2(k).

When $S=1$ the global global phase portrait is:

(37) for $A_{0}<0$;

(50) for $A_{0}>1$ and $C_{2}<0$;

(51) for $0<A_{0}<1$;

(56) for $A_{0}>1$ and $C_{2}=0$;

(57) for $A_{0}=1$;

(58) for $A_{0}>1$ and $C_{2}>0$.

The bifurcation diagram is shown in Figure 2(l).

(K4a) For the family (K4a) the parameter space is $\left(T, A_{0}\right) \in\{-1,0,1\} \times(\mathbb{R} \backslash\{0\})$.

When $T=-1$ the global phase portrait is:

(59) for $A_{0}<0$;

(60) for $0<A_{0}<1$;

(61) for $A_{0}=1$;

(62) for $A_{0}>1$.

When $T=0$ the global phase portrait is: 
(63) for $A_{0}<0$;

(64) for $0<A_{0}<1$;

(65) for $A_{0}=1$;

(66) for $A_{0}>1$.

When $T=1$ the global phase portrait is:

(67) for $A_{0}<0$;

(68) for $0<A_{0}<1$;

(69) for $A_{0}=1$;

(70) for $A_{0}>1$.

(K5a) For the family (K5a) the parameter space is $\left(S, A_{0}, B_{0}\right) \in\{-1,1\} \times(\mathbb{R} \backslash\{0\}) \times \mathbb{R}^{+}$.

Let $C_{3}:=B_{0}^{2}-4 A_{0} S$

When $S=-1$ the global phase portrait is:

(71) for $A_{0}<0$ and $C_{3}<0$;

(72) for $A_{0}<0$ and $C_{3}=0$;

(73) for $A_{0}<0$ and $C_{3}>0$;

(74) $0<A_{0}<1$;

(75) for $A_{0}=1$;

(76) for $A_{0}>1$;

The bifurcation diagram is shown in Figure $2(m)$.

When $S=1$ the global phase portrait is:

(71) for $A_{0}>1$ and $C_{3}<0$;

(72) for $A_{0}>1$ and $C_{3}=0$;

(73) for $A_{0}>1$ and $C_{3}>0$;

(77) for $A_{0}<0$;

(78) for $0<A_{0}<1$ and $C_{3}>0$;

(79) for $A_{0}=1$ and $C_{3}>0$;

(80) for $A_{0}=1$ and $C_{3}=0$;

(81) for $0<A_{0}<1$ and $C_{3}=0$;

(82) for $A_{0}=1$ and $C_{3}<0$;

(83) for $0<A_{0}<1$ and $C_{3}<0$;

The bifurcation diagram is shown in Figure 2(n).

(K5b) For the family (K5b) the parameter space is $\left(S, A_{0}\right) \in\{-1,1\} \times(\mathbb{R} \backslash\{0\})$.

When $S=-1$ the global phase portrait is:

(71) for $A_{0}<0$;

(74) for $0<A_{0}<1$;

(75) for $A_{0}=1$;

(76) for $A_{0}>1$.

When $S=1$ the global phase portrait is:

(71) for $A_{0}>1$;

(82) for $A_{0}=1$;

(83) for $0<A_{0}<1$;

(84) for $A_{0}<0$.

(K5c) For the family $(\mathrm{K} 5 \mathrm{c})$ the parameter space is $\left(S, A_{0}\right) \in\{-1,1\} \times(\mathbb{R} \backslash\{0\})$.

When $S=-1$ the global phase portrait is:

(35) for $A_{0}>1$;

(53) for $A_{0}=1$;

(54) for $0<A_{0}<1$;

(55) for $A_{0}<0$.

When $S=1$ the global phase portrait is:

(37) for $A_{0}<0$;

(51) for $0<A_{0}<1$;

(57) for $A_{0}=1$;

(58) for $A_{0}>1$. 
Remark 1. The families appearing in Proposition 2 do not have limit cycles. Here we provide an explanation of this fact.

Quadratic systems having a straight line can have at most one limit cycle. Moreover, this limit cycle must surround a focus. In our families $x=0$ is always invariant, hence the system has at most one limit cycle. Moreover, this limit cycle must surround a focus not on $x=0$, and hence on $y=0$ (because $\dot{x}=x y$ ). Cases with the symmetries $S_{a}$ or $S_{b}$ cannot have limit cycles, the former because the symmetry would make appear two limit cycles, the latter because no foci can exist on $y=0$. So it remains to explore the cases with the symmetry $S_{c}$.

The only situation where a focus (actually two because of the symmetry) appears is in family (K1c), concretely in phase portraits (25)-(31). In all these phase portraits each focus is the $\alpha$-or $\omega$-limit of a separatrix of a saddle, hence no limit cycles apply here.

The paper is organized as follows. Section 2 provides some classical results about phase portraits. Section 3 is devoted to the proof of Propositions 1 and 2. Finally we prove Theorem 4 in section 4 .

\section{Preliminary Results}

In this section we introduce the basic definitions, notations and results that we need for the analysis of the local phase portraits of the finite and infinite singular points of the quadratic differential systems and afterwards we define the Poincare compactification. The results of subsections 2.1 and 2.3 can be found in [5]. The results of subsection 2.2 can be found in $[6]$.

2.1. Singular points. Consider an analytic planar differential system $\dot{x}=P(x, y), \dot{y}=$ $Q(x, y)$ and its associated vector field $X=(P, Q)$. A point $p \in \mathbb{R}^{2}$ is a singular point of $X$ if $P(p)=Q(p)=0$. We define, for a singular point $p \in \mathbb{R}^{2}, \Delta=P_{x}(p) Q_{y}(p)-P_{y}(p) Q_{x}(p) \in \mathbb{R}$ and $T=P_{x}(p)+Q_{y}(p) \in \mathbb{R}$. They correspond, respectively, to the determinant and the trace of the Jacobian matrix $D X(p)$.

The singular point $p$ is non-degenerated if $\Delta \neq 0$. It is degenerated otherwise. Then, $p$ is an isolated singular point. Moreover $p$ is a saddle if $\Delta<0$, a node if $T^{2} \geq 4 \Delta>0$ (stable if $T<0$, unstable if $T>0$ ), a focus if $4 \Delta>T^{2}>0$ (stable if $T<0$, unstable if $T>0$ ), and either a weak focus or a center if $T=0<\Delta$; for more details see [3].

The singular point $p$ is called hyperbolic if the two eigenvalues of the Jacobian matrix $D X(p)$ have nonzero real part. So the hyperbolic singular points are the non-degenerate ones except the weak foci and the centers.

A degenerate singular point $p$ such that $T \neq 0$ is called semi-hyperbolic, and $p$ is isolated in the set of all singular points. Next we summarize the results on semi-hyperbolic singular points, see Theorem 65 of [3].

Proposition 5. Let $(0,0)$ be an isolated singular point of the vector field $(F(x, y), y+$ $G(x, y))$, where $F$ and $G$ are analytic functions in a neighborhood of the origin starting at least with quadratic terms in $x$ and $y$. Let $y=g(x)$ be the solution of the equation $y+G(x, y)=0$ in a neighborhood of $(0,0)$. Assume that $f(x)=F(x, g(x))=\mu x^{m}+\cdots$, where $m \geq 2$ and $\mu \neq 0$. If $m$ is odd then the origin is either an unstable node or a saddle depending on whether $\mu>0$ or $\mu<0$, respectively. If $m$ is even then $(0,0)$ is a saddle-node, i.e. the singular point is formed by the union of two hyperbolic sectors with one parabolic sector.

The singular points which are non-degenerate or semi-hyperbolic are called elementary.

When $\Delta=T=0$ but the Jacobian matrix at $p$ is not the zero matrix and $p$ is isolated in the set of all singular points, we say that $p$ is nilpotent. Next we summarize some results on nilpotent singular points (see Theorems 66 and 67 and the simplified scheme of section 22.3 of $[3])$. 


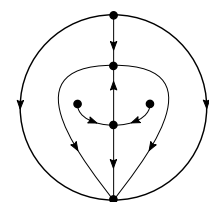

(1)

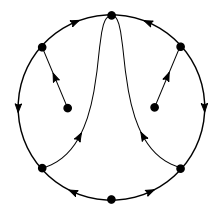

(7)

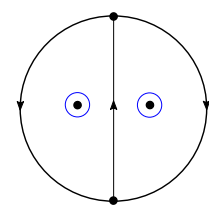

(13)

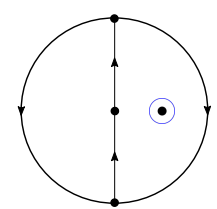

(19)

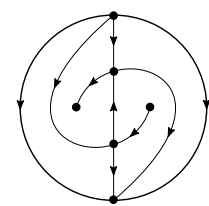

(25)

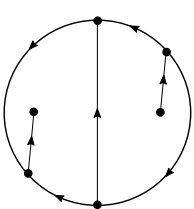

(31)

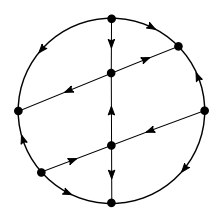

(37)

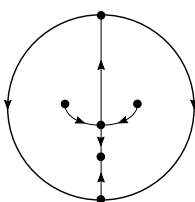

(2)

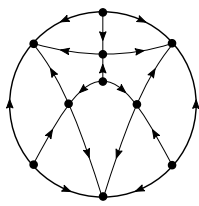

(8)

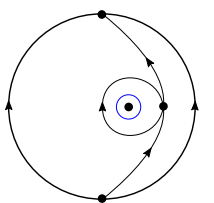

(14)

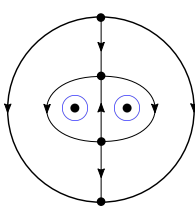

(20)

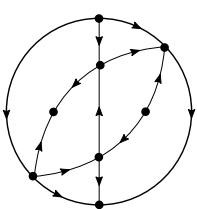

(26)

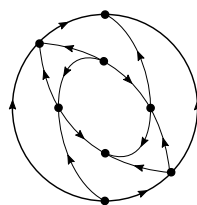

(32)

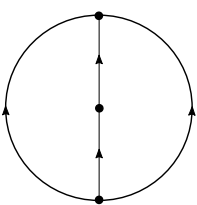

(38)

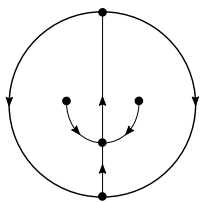

(3)

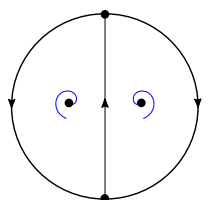

(4)

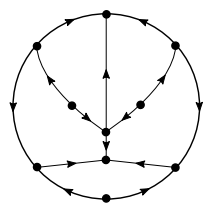

(5)

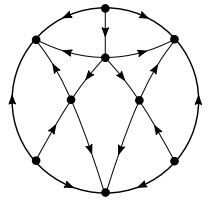

(9)

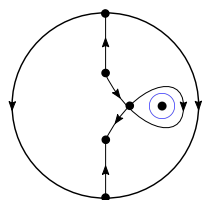

(15)

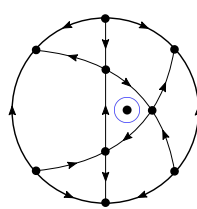

(21)

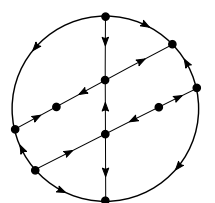

(27)

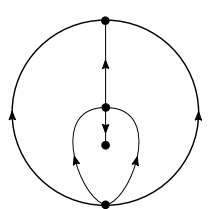

(33)

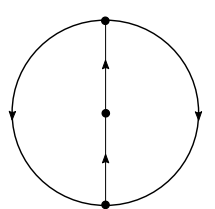

(39)

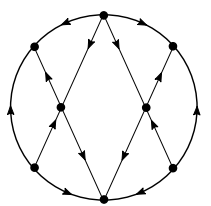

(10)

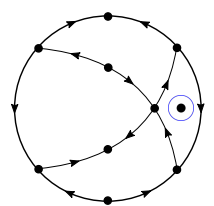

(16)

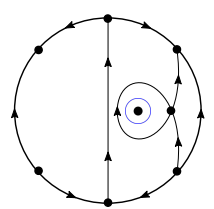

(22)

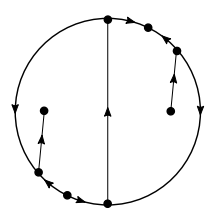

(28)

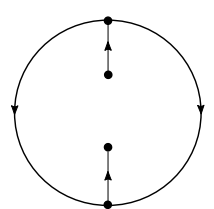

(34)

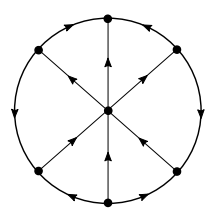

(40)

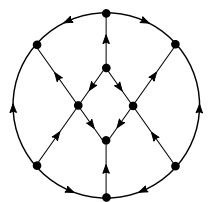

(11)

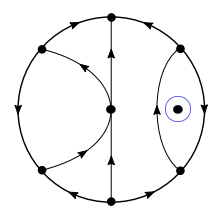

(17)

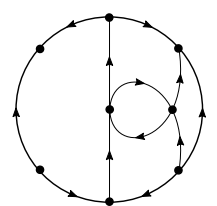

(23)

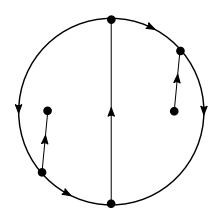

(29)

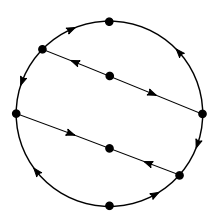

(35)

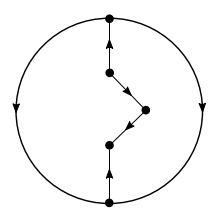

(41)

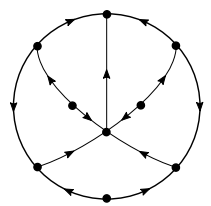

(6)

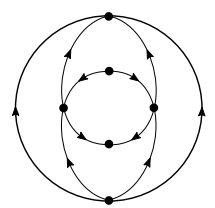

(12)

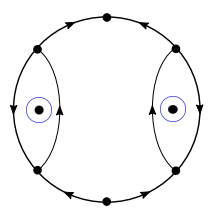

(18)

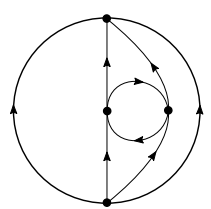

(24)

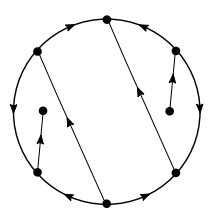

(30)

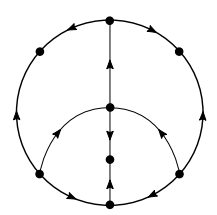

(36)

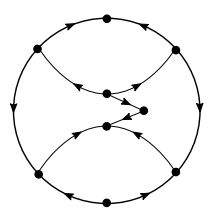

(42)

Figure 1. Phase portraits of the differential systems appearing in Theorems 3 and $4(\mathrm{I})$.

Proposition 6. Let $(0,0)$ be an isolated singular point of the vector field $(y+F(x, y)$, $G(x, y))$, where $F$ and $G$ are analytic functions in a neighborhood of the origin starting at least with quadratic terms in $x$ and $y$. Let $y=f(x)$ be the solution of the equation $y+F(x, y)=0$ in a neighborhood of $(0,0)$. Assume that $G(x, f(x))=K x^{\kappa}+\cdots$ and $\Phi(x) \equiv(\partial F / \partial x+\partial G / \partial y)(x, f(x))=L x^{\lambda}+\cdots$, with $K \neq 0, \kappa \geq 2$ and $\lambda \geq 1$. The following statements hold.

(i) If $\kappa$ is even and 


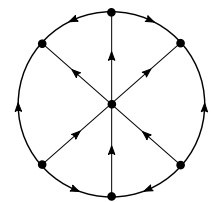

(43)

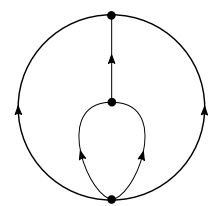

(49)

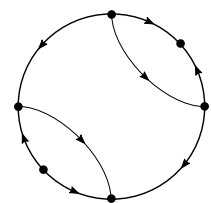

(55)

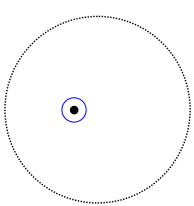

(61)

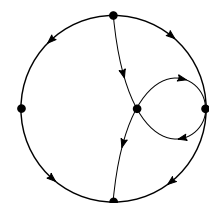

(67)

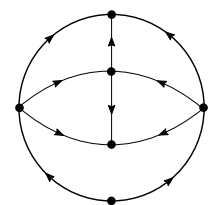

(73)

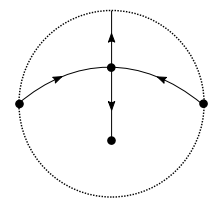

(79)

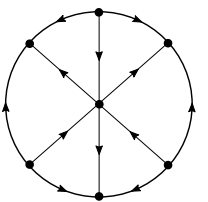

(44)

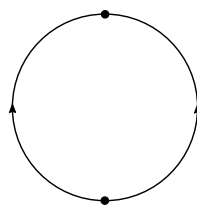

(50)

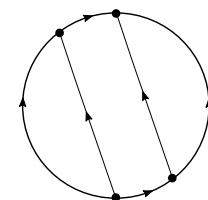

(56)

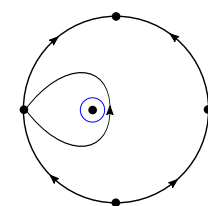

(62)

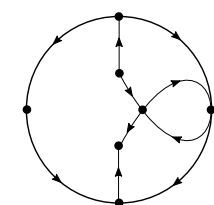

(68)

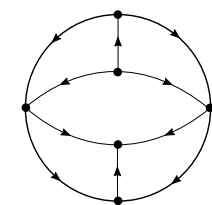

(74)

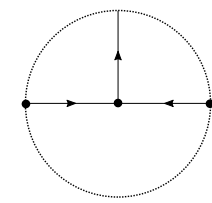

(80)

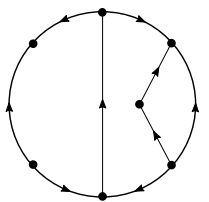

(45)

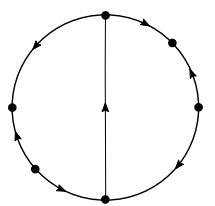

(51)

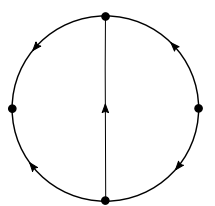

(57)

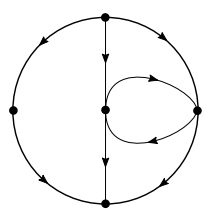

(63)

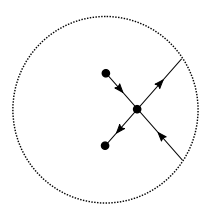

(69)

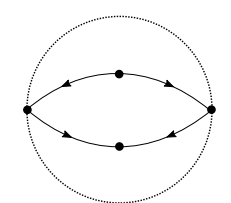

(75)

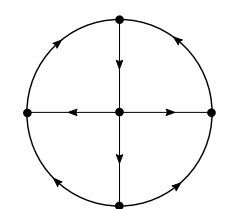

(81)

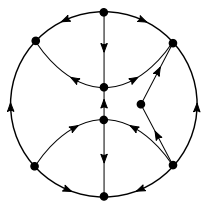

(46)

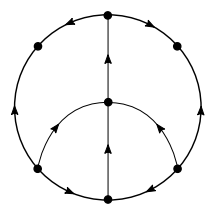

(52)

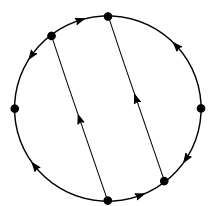

(58)

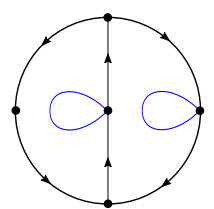

(64)

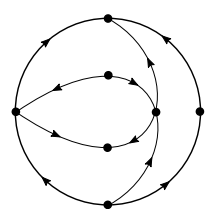

(70)

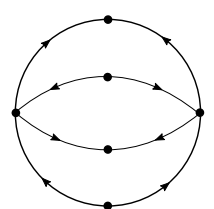

(76)

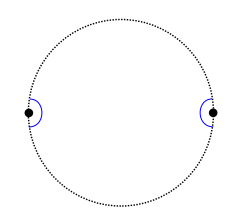

(82)

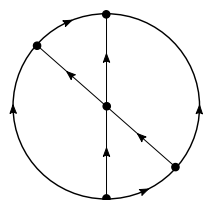

(47)

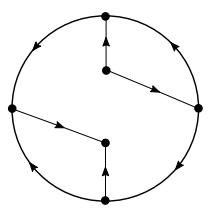

(53)

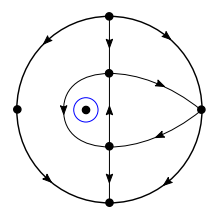

(59)

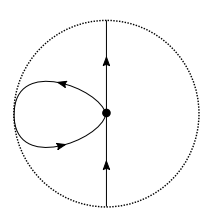

(65)

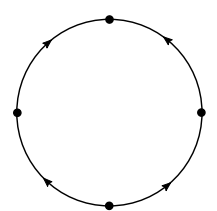

(71)

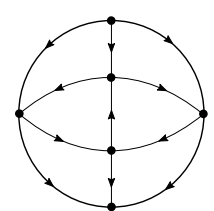

(77)

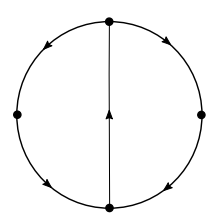

(83)

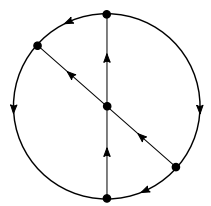

(48)

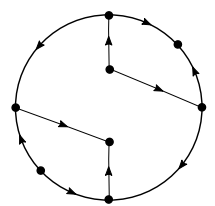

(54)

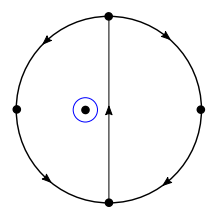

(60)

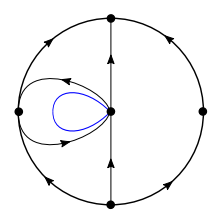

(66)

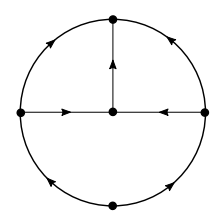

(72)

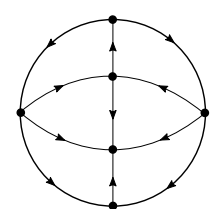

(78)

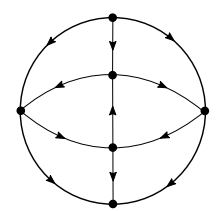

(84)

Figure 1. Phase portraits of the differential systems appearing in Theorems 3 and 4 (and II).

(a) $\kappa>2 \lambda+1$ then the origin is a saddle-node.

(b) $\kappa<2 \lambda+1$ or $\Phi \equiv 0$ then the origin is a cusp, i.e. a singular point formed by the union of two hyperbolic sectors.

(ii) If $\kappa$ is odd and $K>0$ then the origin is a saddle.

(iii) If $\kappa$ is odd, $K<0$ and

(a) $\lambda$ even, $\kappa=2 \lambda+1$ and $L^{2}+4 K(\lambda+1) \geq 0$, or $\lambda$ even and $\kappa>2 \lambda+1$ then the origin is a stable (unstable) node if $L<0(L>0)$. 


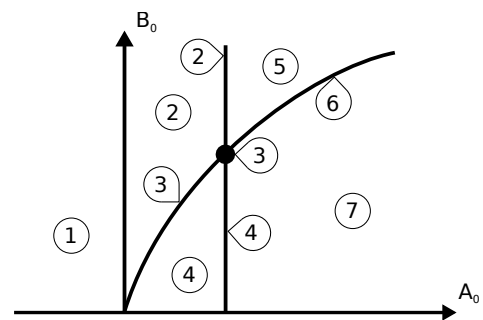

(a)

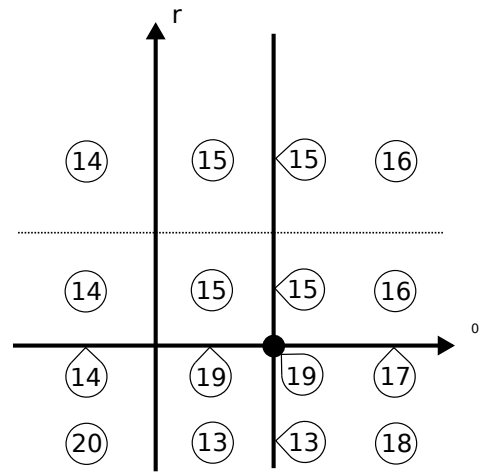

(c)

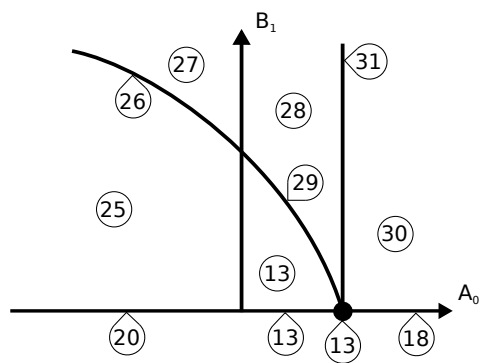

(e)

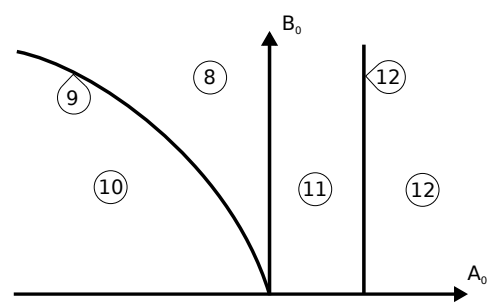

(b)

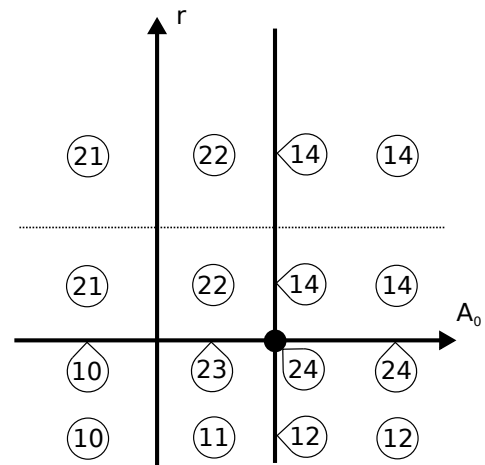

(d)

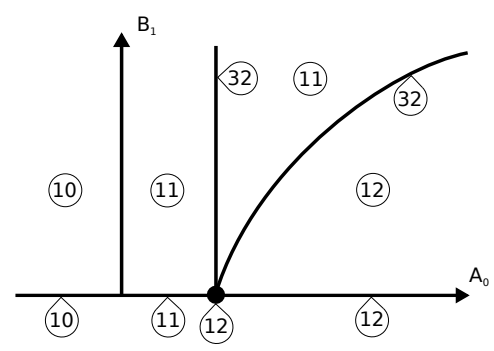

(f)

FIGURE 2. Bifurcation diagrams of the families appearing in Theorems 3 and 4 (I): (a) (K1a) in the case $S=-1$; (b) (K1a) in the case $S=1$; (c) (K1b) in the case $S=-1 ;(\mathrm{d})(\mathrm{K} 1 \mathrm{~b})$ in the case $S=1 ;(\mathrm{e})(\mathrm{K} 1 \mathrm{c})$ in the case $S=-1$; (f) (K1c) in the case $S=1$.

(b) $\lambda$ odd, $\kappa=2 \lambda+1$ and $L^{2}+4 K(\lambda+1) \geq 0$, or $\lambda$ odd and $\kappa>2 \lambda+1$ then the origin is an elliptic saddle, i.e. a singular point formed by the union of one hyperbolic sector and one elliptic sector.

(c) $\kappa=2 \lambda+1$ and $L^{2}+4 K(\lambda+1)<0$, or $\kappa<2 \lambda+1$ then the origin is a focus or a center, and if $\Phi(x) \equiv 0$ then the origin is a center.

Finally if the Jacobian matrix at the singular point $p$ is identically zero and $p$ is isolated inside the set of all singular points then we say that $p$ is linearly zero. The study of its local phase portrait needs a special treatment: the directional blow-ups, see for more details $[2,4]$. But if a quadratic vector field has a finite linearly zero singular point then it is equivalent to a homogeneous quadratic differential system doing if necessary a translation of the linearly zero singular point to the origin, and the global phase portraits of the quadratic homogeneous vector fields are well known, see [13].

The definitions of hyperbolic, parabolic and elliptic sectors near a singular point can be found in [3]. Roughly speaking, in a hyperbolic sector there are curves through points of the 


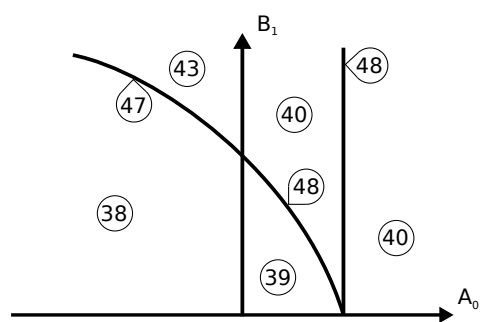

(g)

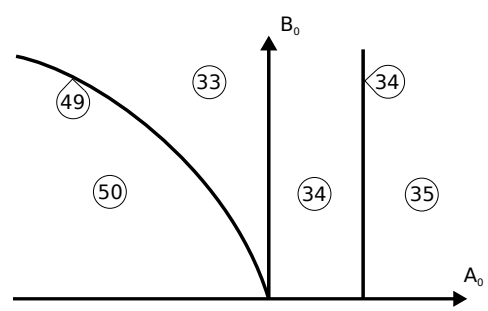

(i)

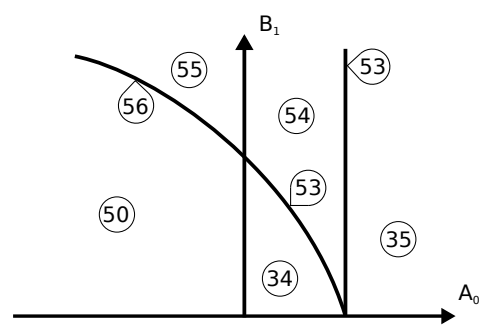

$(\mathrm{k})$

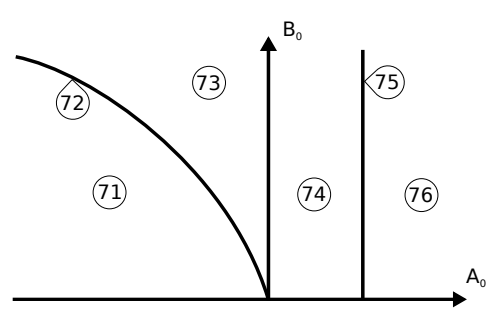

$(\mathrm{m})$

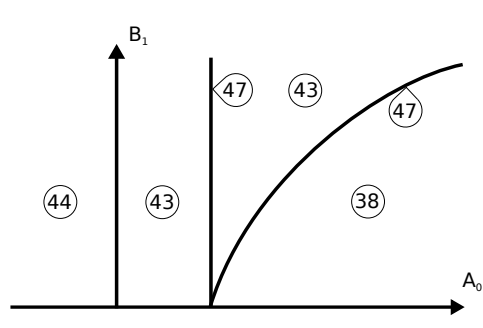

(h)

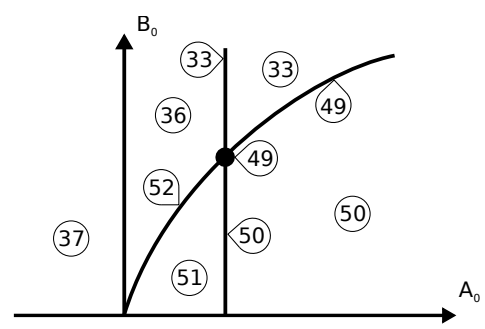

(j)

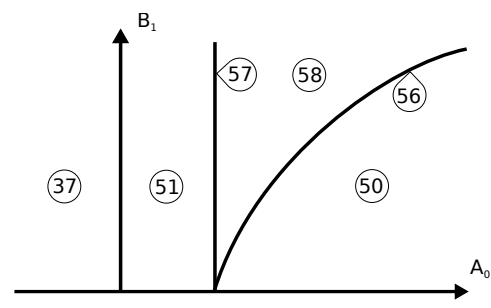

(l)

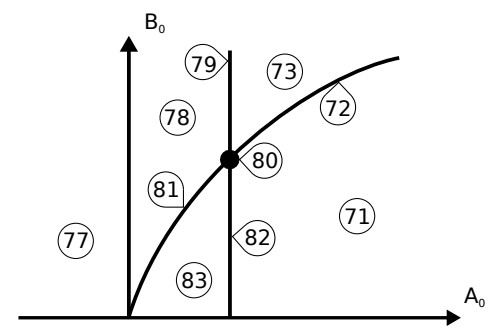

(n)

FiguRE 2. Bifurcation diagrams of the families appearing in Theorems 3 and 4 (and II): (g) (K2c) in the case $S=-1$; (h) (K2c) in the case $S=1$; (i) (K3a) in the case $S=-1$; (j) (K3a) in the case $S=1$; (k) (K3c) in the case $S=-1$; (l) (K3c) in the case $S=1 ;(\mathrm{m})(\mathrm{K} 5 \mathrm{a})$ in the case $S=-1$; (n) (K5a) in the case $S=1$.

sector which leave the sector with both increasing and decreasing time. A sector such that all curves in a sufficiently small neighborhood of the singular point tend to it as $t \rightarrow+\infty$ $(t \rightarrow-\infty)$ and leave the sector as $t \rightarrow-\infty(t \rightarrow+\infty)$ is known as a parabolic sector. Finally, a sector containing loops, and moreover only nested loops, is known as an elliptic sector.

The number of elliptic sectors and the number of hyperbolic sectors in a neighbourhood of a singular point are denoted by $\mathbf{e}$ and $\mathbf{h}$, respectively. The rest of the sectors are parabolic. 
The index of a singular point $p$ is defined as $i(p)=(\mathbf{e}-\mathbf{h}) / 2+1 \in \mathbb{Z}$. For a proof of this formula see [3].

Next we state a result of [9] that allows to distinguish between a center and a focus for a singular point of a quadratic differential system having pure imaginary eigenvalues.

Theorem 7. Consider the quadratic differential system

$$
\dot{x}=-y+a_{20} x^{2}+a_{11} x y+a_{02} y^{2}, \quad \dot{y}=x+b_{20} x^{2}+b_{11} x y+b_{02} y^{2} .
$$

Let

$$
\omega_{1}=A \alpha-B \beta, \quad \omega_{2}=\gamma\left(5(A \beta+B \alpha)-\alpha^{2}-\beta^{2}\right), \quad \omega_{3}=\gamma \delta(A \beta+B \alpha),
$$

where

$$
\begin{aligned}
A & =a_{20}+a_{02}, \quad B=b_{20}+b_{02}, \quad \alpha=a_{11}+2 b_{02}, \quad \beta=b_{11}+2 a_{20}, \\
\gamma & =b_{20} A^{3}-\left(a_{20}-b_{11}\right) A^{2} B+\left(b_{02}-a_{11}\right) A B^{2}-a_{02} B^{3}, \\
\delta & =a_{02}^{2}+b_{20}^{2}+a_{02} A+b_{20} B .
\end{aligned}
$$

Then the following statements hold:

(1) If $\omega_{1}=\omega_{2}=\omega_{3}=0$ then the origin is a center.

(2) If there exists $k \in\{1,2,3\}$ such that $\omega_{j}=0$ for all $1 \leq j<k$ and $\omega_{k} \neq 0$, then the origin is a focus of order $k$. The stability of this focus is given by the sign of $\omega_{k} \neq 0$ : when $\omega_{k}<0$ the focus is stable and when $\omega_{k}>0$ the focus is unstable.

2.2. Separatrices and canonical regions. Consider the planar differential system $\dot{x}=$ $P(x, y), \dot{y}=Q(x, y)$, where $P, Q \in \mathcal{C}^{r}, r \geq 1$. For this differential system the following three properties are well-known, see for more details [12]:

(i) For all $p \in U$ there exists an open interval $I_{p} \subseteq \mathbb{R}$ where the unique maximal solution $\varphi_{p}: I_{p} \rightarrow U$ of the system such that $\varphi_{p}(0)=p$ is defined.

(ii) If $q=\varphi_{p}(t)$ and $t \in I_{p}$ then $I_{q}=I_{p}-t=\left\{r-t: r \in I_{p}\right\}$ and $\varphi_{q}(s)=\varphi_{p}(t+s)$ for all $s \in I_{q}$.

(iii) The set $D=\left\{(t, p): p \in U, t \in I_{p}\right\}$ is open in $\mathbb{R}^{3}$ and the map $\varphi: D \rightarrow U$ defined by $\varphi(t, p)=\varphi_{p}(t)$ is $\mathcal{C}^{r}$.

The map $\varphi: D \rightarrow U$ is a local flow of class $\mathcal{C}^{r}$ on $U$ associated to the system. It verifies:

(1) $\varphi(0, p)=p$ for all $p \in U$;

(2) $\varphi(t, \varphi(s, p))=\varphi(t+s, p)$ for all $p \in U$ and for all $s$ and $t$ such that $s, t+s \in I_{p}$;

(3) $\varphi_{p}(-t)=\varphi_{p}^{-1}(t)$ for all $p \in U$ such that $t,-t \in I_{p}$.

We consider $\mathcal{C}^{r}$-local flows on $\mathbb{R}^{2}$, with $r \geq 0$. Of course when $r=0$ the flow is only continuous. Two such flows $\varphi$ and $\varphi^{\prime}$ are $\mathcal{C}^{k}$-equivalent, with $k \geq 0$, if there exists a $\mathcal{C}^{k}$ diffeomorphism that brings orbits of $\varphi$ onto orbits of $\varphi^{\prime}$ preserving sense (but not necessarily the parametrization).

Let $\varphi$ be a $\mathcal{C}^{r}$-local flow with $r \geq 0$ on $\mathbb{R}^{2}$. We say that $\varphi$ is $\mathcal{C}^{k}$-parallel if it is $\mathcal{C}^{k}$-equivalent to one of the following flows:

(i) $\mathbb{R}^{2}$ with the flow defined by $x^{\prime}=1, y^{\prime}=0$.

(ii) $\mathbb{R}^{2} \backslash\{0\}$ with the flow defined (in polar coordinates) by $r^{\prime}=0, \theta^{\prime}=1$.

(iii) $\mathbb{R}^{2} \backslash\{0\}$ with the flow defined by $r^{\prime}=r, \theta=0$.

We call these flows strip, annular and spiral, respectively.

Let $p \in \mathbb{R}^{2}$. We denote by $\gamma(p)$ the orbit of the flow $\varphi$ through $p$, i.e. $\gamma(p)=\left\{\varphi_{p}(t)\right.$ : $\left.t \in I_{p}\right\}$. The positive semiorbit of $p$ is $\gamma^{+}(p)=\left\{\varphi_{p}(t): t \in I_{p}, t \geq 0\right\}$. In a similar way we define the negative semiorbit $\gamma^{-}(p)$ of $p$.

We define the $\alpha$-limit and the $\omega$-limit of $p$ as $\left(\gamma^{ \pm}(p)\right)$ and let

$$
\alpha(p)=\operatorname{cl}\left(\gamma^{-}(p)\right)-\gamma^{-}(p), \omega(p)=\operatorname{cl}\left(\gamma^{+}(p)\right)-\gamma^{+}(p),
$$

respectively. Here, as usual, cl denotes the closure. 
Let $\gamma(p)$ be an orbit of the flow $\varphi$. A parallel neighborhood of the orbit $\gamma(p)$ is an open neighborhood $N$ of $\gamma(p)$ such that $\varphi$ is $\mathcal{C}^{k}$-equivalent in $N$ to a parallel flow for some $k \geq 0$.

We say that $\gamma(p)$ is a separatrix of $\varphi$ if it is not contained in a parallel neighborhood $N$ satisfying the following two assumptions:

(i) for any $q \in N, \alpha(q)=\alpha(p)$ and $\omega(q)=\omega(p)$;

(ii) $\operatorname{cl}(N) \backslash N$ consists of $\alpha(p), \omega(p)$ and exactly two orbits $\gamma(a), \gamma(b)$ of $\varphi$, with $\alpha(a)=$ $\alpha(p)=\alpha(b)$ and $\omega(a)=\omega(p)=\omega(b)$.

We denote by $\Sigma$ the union of all separatrices of $\varphi$. $\Sigma$ is a closed invariant subset of $\mathbb{R}^{2}$. A component of the complement of $\Sigma$ in $\mathbb{R}^{2}$, with the restricted flow, is a canonical region of $\varphi$.

The following lemma can be found in [10].

Lemma 8. Every canonical region of a local flow $\varphi$ on $\mathbb{R}^{2}$ is $\mathcal{C}^{0}$-parallel.

2.3. The Poincaré compactification. Let $X$ be a real planar polynomial vector field of degree $n$. The Poincaré compactified vector field $p(X)$ corresponding to $X$ is an analytic vector field induced on $\mathbb{S}^{2}$ as follows (see for instance [8]). Let $\mathbb{S}^{2}=\left\{y=\left(y_{1}, y_{2}, y_{3}\right) \in \mathbb{R}^{3}\right.$ : $\left.y_{1}^{2}+y_{2}^{2}+y_{3}^{2}=1\right\}$ (the Poincaré sphere) and $T_{y} \mathbb{S}^{2}$ be the tangent plane to $\mathbb{S}^{2}$ at a point $y$. Identify $\mathbb{R}^{2}$ with $T_{(0,0,1)} \mathbb{S}^{2}$. Consider the central projection $f: T_{(0,0,1)} \mathbb{S}^{2} \rightarrow \mathbb{S}^{2}$. This map defines two copies of $X$ on $\mathbb{S}^{2}$, one in the northern hemisphere and the other in the southern hemisphere. Denote by $\bar{X}$ the vector field $D f \circ X$ defined on $\mathbb{S}^{2}$ except on its equator $\mathbb{S}^{1}=\left\{y \in \mathbb{S}^{2}: y_{3}=0\right\}$. Clearly $\mathbb{S}^{1}$ is identified to the infinity of $\mathbb{R}^{2}$. Usually, when we talk about the circle of the infinity of $X$ we simply talk about the infinity.

In order to extend $\bar{X}$ to a vector field on $\mathbb{S}^{2}$ (including $\mathbb{S}^{1}$ ) it is necessary for $X$ to satisfy suitable conditions. If $X$ is a real polynomial vector field of degree $n$ then $p(X)$ is the only analytic extension of $y_{3}^{n-1} \bar{X}$ to $\mathbb{S}^{2}$. On $\mathbb{S}^{2} \backslash \mathbb{S}^{1}$ there are two symmetric copies of $X$, and knowing the behavior of $p(X)$ around $\mathbb{S}^{1}$ we know the behavior of $X$ in a neighborhood of the infinity. The Poincare compactification has the property that $\mathbb{S}^{1}$ is invariant under the flow of $p(X)$. The projection of the closed northern hemisphere of $\mathbb{S}^{2}$ on $y_{3}=0$ under $\left(y_{1}, y_{2}, y_{3}\right) \mapsto\left(y_{1}, y_{2}\right)$ is called the Poincare disc, and it is denoted by $\mathbb{D}^{2}$.

Two polynomial vector fields $X$ and $Y$ on $\mathbb{R}^{2}$ are topologically equivalent if there exists a homeomorphism on $\mathbb{S}^{2}$ preserving the infinity $\mathbb{S}^{1}$ and carrying orbits of the flow induced by $p(X)$ into orbits of the flow induced by $p(Y)$.

As $\mathbb{S}^{2}$ is a differentiable manifold, for computing the expression of $p(X)$ we can consider the six local charts $U_{i}=\left\{y \in \mathbb{S}^{2}: y_{i}>0\right\}$, and $V_{i}=\left\{y \in \mathbb{S}^{2}: y_{i}<0\right\}, i=1,2,3$. The diffeomorphisms $F_{i}: U_{i} \rightarrow \mathbb{R}^{2}$ and $G_{i}: V_{i} \rightarrow \mathbb{R}^{2}$ for $i=1,2,3$ are the inverses of the central projections from the planes tangent at the points $(1,0,0),(-1,0,0),(0,1,0),(0,-1,0),(0,0,1)$ and $(0,0,-1)$, respectively. If $z=(u, v)$ is the value of $F_{i}(y)$ or $G_{i}(y)$ for any $i=1,2,3$ (so $z$ represents different things according to the local charts under consideration) then we obtain the following expressions for $p(X)$ :

$$
v^{n} \Delta(z)\left(Q\left(\frac{1}{v}, \frac{u}{v}\right)-u P\left(\frac{1}{v}, \frac{u}{v}\right),-v P\left(\frac{1}{v}, \frac{u}{v}\right)\right)
$$

on $U_{1}$;

$$
v^{n} \Delta(z)\left(P\left(\frac{u}{v}, \frac{1}{v}\right)-u Q\left(\frac{u}{v}, \frac{1}{v}\right),-v Q\left(\frac{u}{v}, \frac{1}{v}\right)\right)
$$

on $U_{2}$; and

$$
\Delta(z)(P(u, v), Q(u, v))
$$

on $U_{3}$, where $\Delta(z)=\left(u^{2}+v^{2}+1\right)^{-\frac{1}{2}(n-1)}$. The expression for $V_{i}$ is the same as that for $U_{i}$ except for a multiplicative factor $(-1)^{n-1}$. In these coordinates, for $i=1,2, v=0$ always denotes the points of $\mathbb{S}^{1}$. We can omit the factor $\Delta(z)$ by scaling the vector field $p(X)$. Thus, the expression of $p(X)$ becomes a polynomial vector field in each local chart. 


\section{Proofs of Propositions 1 And 2}

We prove in this section Propositions 1 and 2. We recall that these results allow us to split the differential system (3) into the thirteen different families that are discussed in Theorems 3 and 4 .

Proof of Proposition 1. By applying the change of variables and time $(x, y) \rightarrow\left(x+\frac{d_{0}}{d_{1}}, B_{2} y\right)$, $\frac{d t}{d \tau}=\frac{B_{2}}{d_{1}}$, system (3) becomes

$$
\begin{aligned}
\dot{x}= & x y, \\
\dot{y}= & \frac{B_{2}^{2}\left(c_{0} d_{1}^{2}-c_{1} d_{0} d_{1}+c_{2} d_{0}^{2}\right)}{d_{1}^{3}}+\frac{B_{2}^{2}\left(c_{1} d_{1}-2 c_{2} d_{0}\right)}{d_{1}^{2}} x+\frac{B_{2}\left(b_{0} d_{1}-b_{1} d_{0}\right)}{d_{1}^{2}} y \\
& +\frac{B_{2}^{2} c_{2}}{d_{1}} x^{2}+\frac{B_{2} b_{1}}{d_{1}} x y+\frac{a_{0}}{d_{1}} y^{2} .
\end{aligned}
$$

We consider first the case $c_{2} \neq 0$, for which system (8) has at most four singular points. Renaming

$$
d_{1}=\frac{a_{0}}{A_{0}}, \quad B_{2}=\sqrt{\frac{a_{0} S}{A_{0} c_{2}}}, \quad b_{1}=B_{1} \sqrt{\frac{a_{0} c_{2}}{A_{0} S}}, \quad b_{0}=\sqrt{\frac{c_{2}}{a_{0} A_{0} S}}\left(A_{0} B_{1} d_{0}+a_{0} B_{0}\right),
$$

we get

$$
\begin{aligned}
& \dot{x}=x y, \\
& \dot{y}=\frac{S\left(A_{0}^{2} c_{2} d_{0}^{2}-A_{0} a_{0} c_{1} d_{0}+a_{2}^{2} c_{0}\right)}{a_{0}^{2} c_{2}}+\frac{S\left(a_{0} c_{1}-2 A_{0} c_{2} d_{0}\right)}{a_{0} c_{2}} x+B_{0} y+S x^{2}+B_{1} x y+A_{0} y^{2} .
\end{aligned}
$$

We focus on the singular points on $y=0$. Let $\Delta:=c_{1}^{2}-4 c_{0} c_{2}$ be the discriminant of $\left.\dot{y}\right|_{y=0}$. If $\Delta>0$ then there are two different real singular points on $y=0$, say $\left(r_{1}, 0\right)$ and $\left(r_{2}, 0\right)$; after renaming

$$
c_{0}=\frac{c_{2}\left(A_{0} d_{0}-a_{0} r_{1}\right)\left(A_{0} d_{0}-a_{0} r_{2}\right)}{a_{0}^{2}}, \quad c_{1}=\frac{c_{2}\left(2 A_{0} d_{0}-a_{0}\left(r_{1}+r_{2}\right)\right)}{a_{0}},
$$

system (9) leads to the family (K1).

If $\Delta=0$ then there is a double real singular point on $y=0$, say $(r, 0)$; after renaming

$$
c_{0}=\frac{c_{2}\left(A_{0} d_{0}-a_{0} r\right)^{2}}{a_{0}^{2}}, \quad c_{1}=\frac{2 c_{2}\left(A_{0} d_{0}-a_{0} r\right)}{a_{0}},
$$

system (9) leads to the family (K2).

If $\Delta<0$ then there are two complex conjugate singular points on $y=0$; after renaming

$$
c_{0}=\frac{c_{2}\left(A_{0} d_{0}-a a_{0}\right)^{2}+a_{0}^{2} b c_{2}}{a_{0}^{2}}, \quad c_{1}=\frac{2 c_{2}\left(A_{0} d_{0}-a_{0} a\right)}{a_{0}},
$$

where $\left.\dot{y}\right|_{y=0}=(x-a)^{2}+b^{2}$, with $b>0$, system (9) leads to the family (K3).

Now we consider the case $c_{2}=0$. If $c_{1} \neq 0$ then applying the change of variables and time $(x, y) \rightarrow\left(\frac{B_{2}^{2} c_{1}}{d_{1}} x+\frac{B_{2}^{2} c_{1} d_{0}}{d_{1}^{2}}, B_{2} y\right), \frac{d t}{d \tau}=\frac{B_{2}}{d_{1}}$, system (3) becomes

$$
\dot{x}=x y, \quad \dot{y}=\frac{B_{2}^{2}\left(c_{0} d_{1}-c_{1} d_{0}\right)}{d_{1}^{2}}+x+\frac{B_{2}\left(b_{0} d_{1}-b_{1} d_{0}\right)}{d_{1}^{2}} y+\frac{b_{1}}{B_{2} c_{1}} x y+\frac{a_{0}}{d_{1}} y^{2} .
$$

Renaming

with

$$
d_{1}=\frac{a_{0}}{A_{0}}, \quad b_{0}=\frac{A_{0}^{2} B_{1} B_{2}^{2} c_{1} d_{0}+a_{0}^{2} B_{0}}{a_{0} A_{0} B_{2}}, \quad b_{1}=B_{1} B_{2} c_{1}
$$

$$
B_{2}=a_{0} \sqrt{\frac{T}{A_{0}\left(A_{0} c_{1} d_{0}-a_{0} c_{0}\right)}} \quad \text { and } \quad T \in\{-1,0,1\},
$$


the above system becomes the family (K4).

If $c_{1}=0$ then, applying the change of variables and time $(x, y) \rightarrow\left(A_{1} x+\frac{A_{1} d_{0}}{d_{1}}, B_{2} y\right)$, $\frac{d t}{d \tau}=\frac{B_{2}}{d_{1}}$, system (3) becomes

Renaming

$$
\dot{x}=x y, \quad \dot{y}=\frac{B_{2}^{2} c_{0}}{d_{1}}+\frac{B_{2}\left(b_{0} d_{1}-b_{1} d_{0}\right)}{d_{1}^{2}} y+\frac{b_{1} B_{2}}{A_{1} d_{1}} x y+\frac{a_{0}}{d_{1}} y^{2} .
$$

with

$$
d_{1}=\frac{a_{0}}{A_{0}}, \quad b_{0}=\frac{A_{0} A_{1} B_{1} d_{0}+a_{0} B_{0}}{A_{0} B_{2}}, \quad b_{1}=\frac{a_{0} A_{1} B_{1}}{A_{0} B_{2}}
$$

$$
B_{2}=\sqrt{\frac{a_{0} S}{A_{0} c_{0}}} \quad \text { and } \quad S \in\{1,-1\},
$$

the above system becomes the family (K5). Then the proposition follows.

Proof of Proposition 2. The proof follows by applying the symmetries $\left(S_{a}\right),\left(S_{b}\right)$ and $\left(S_{c}\right)$ given in (4) and possibly the change of sign of the independent variable $t \mapsto-t$ to the families given in Proposition 1.

Applying $\left(S_{a}\right)$ to the family (K1) we obtain $-\dot{x}=-x y$ and $\dot{y}=S\left(-x-r_{1}\right)\left(-x-r_{2}\right)+$ $\left(B_{0}-B_{1} x\right) y+A_{0} y^{2}$. So we get again (K1) if and only if $r_{1}+r_{2}=0$ and $B_{1}=0$. (K1a) is obtained after additional affine changes which allow us to consider $B_{0}>0$ and to take $r_{1}=-r_{2}=1$.

Applying $\left(S_{b}\right)$ to the family (K1) we obtain $\dot{x}=-x y$ and $-\dot{y}=S\left(x-r_{1}\right)\left(x-r_{2}\right)-\left(B_{0}+\right.$ $\left.B_{1} x\right) y+A_{0} y^{2}$. We need a change of time $t \mapsto-t$ and $B_{0}=B_{1}=0$ to obtain again (K1). Moreover, after additional affine changes we know that we can take $r_{1}=1$ and $r_{2}=r \neq 1$. We get (K1b).

Finally, applying $\left(S_{c}\right)$ to the family (K1) we obtain $\dot{x}=x y$ and $-\dot{y}=S\left(-x-r_{1}\right)(-x-$ $\left.r_{2}\right)-\left(B_{0}-B_{1} x\right) y+A_{0} y^{2}$. We need to apply a change of time $t \mapsto-t$ and set $r_{1}+r_{2}=0$ and $B_{0}=0$. Additionally we can take $r_{1}=-r_{2}=1$ and $B_{1} \geq 0$. We get (K1c).

The other families are obtained in an analogous way. The proposition follows.

\section{Proof of Theorem 4}

We recall that Theorem 3 follows after Theorem 4. Following Proposition 2, we prove Theorem 4 separately for the thirteen different families.

Proof of Theorem $4($ K1a). We deal with the differential system

$$
\dot{x}=x y, \quad \dot{y}=S\left(x^{2}-1\right)+B_{0} y+A_{0} y^{2},
$$

where $S \in\{-1,1\}, A_{0} \neq 0$, and $B_{0}>0$.

The finite singular points of system $(10)$ are $( \pm 1,0)$ and $\left(0,\left(-B_{0} \pm \sqrt{B_{0}^{2}+4 A_{0} S}\right) / 2 A_{0}\right)$. The eigenvalues of the singular points $( \pm 1,0)$ are $\left(B_{0} \pm \sqrt{B_{0}^{2}+8 S}\right) / 2$. If $S=1$ then both singular points are saddles. If $S=-1$ then we have two cases: if $B_{0} \geq 2 \sqrt{2}$ then the singular points are unstable nodes whereas if $B_{0}<2 \sqrt{2}$ then the singular points are unstable foci.

Let $C_{1}:=B_{0}^{2}+4 A_{0} S$. Then the other two singular points are $\left(0,\left(-B_{0} \pm \sqrt{C_{1}}\right) / 2 A_{0}\right)$ and they are real only if $C_{1} \geq 0$. Suppose first that $C_{1}>0$. The eigenvalues of the singular point $\left(0,\left(-B_{0}+\sqrt{C_{1}}\right) / 2 A_{0}\right)$ are $\sqrt{C_{1}}$ and $\left(-B_{0}+\sqrt{C_{1}}\right) / 2 A_{0}$. Direct computations show that if $S=1$ then the point is a unstable node and if $S=-1$ then it is a saddle point. On the other hand, the point $\left(0,\left(-B_{0}-\sqrt{C_{1}}\right) / 2 A_{0}\right)$ has eigenvalues $-\sqrt{C_{1}}$, and $\left(-B_{0}-\sqrt{C_{1}}\right) / 2 A_{0}$. Then if $A_{0}<0$ it is a saddle whereas if $A_{0}>0$ it is a stable node.

If $C_{1}=0$ then the two points become the singular point $\left(0,2 S / B_{0}\right)$ which is semihyperbolic. We move the point to the origin and we apply the change of variables and time $(x, y) \rightarrow(y, x), \frac{d t}{d \tau}=\frac{B_{0}}{2 S}$, to bring the system to the form given in Proposition 5. So, $F(x, f(x))=-\left(B_{0}^{3} / 8 S^{2}\right) x^{2}+\ldots$ and the singular point is saddle-node. 
According to expression (7), system (10) at infinity can be studied from the system

$$
\dot{u}=-u\left(S u^{2}-S v^{2}+B_{0} v+A_{0}-1\right), \quad \dot{v}=-v\left(S u^{2}-S v^{2}+B_{0} v+A_{0}\right),
$$

on $U_{2}$. We do not need to study the expression (6) because all the infinite singular points can be studied from the above differential system. We have three infinite singular points: $(0,0)$ and $\left( \pm \sqrt{\left(1-A_{0}\right) / S}, 0\right)$. The origin on $U_{2}$ has eigenvalues $-A_{0}, 1-A_{0}$, thus it is an unstable node if $A_{0}<0$, a saddle if $0<A_{0}<1$ or a stable node if $A_{0}>1$. When $A_{0}=1$ we apply Proposition 5 because the point is a semi-hyperbolic point. So we have $F(u, g(u))=f(u)=S u^{3}+\cdots$ and therefore if $S=-1$ the point is a saddle and if $S=1$ the point is a stable node, because of a change of time before applying Proposition 5 . The other two infinite singular points, $\left( \pm \sqrt{\left(1-A_{0}\right) / S}, 0\right)$, only exist if $S\left(1-A_{0}\right)>0$. Both of them have eigenvalues $-1,-2\left(1-A_{0}\right)$. Thus, for $S=1$ and $A_{0}<1$ the points are stable nodes and for $S=-1$ and $A_{0}>1$ the points are saddles. Statement (K1a) of Theorem 4 follows.

Table 1 summarizes the different behaviors of the singular points depending on the values of the parameters.

\begin{tabular}{|c|c|c|}
\hline Singular point & Conditions & Type of point \\
\hline \hline \multirow{2}{*}{$( \pm 1,0)$} & $S=1$ & Saddles \\
\cline { 2 - 3 } & $S=-1, B_{0}<2 \sqrt{2}$ & Unstable Foci \\
\cline { 2 - 3 }$\left(0, \frac{-B_{0}+\sqrt{C_{1}}}{2 A_{0}}\right)$ & $S=-1, B_{0} \geq 2 \sqrt{2}$ & Unstable Nodes \\
\cline { 2 - 3 } & $S=1, C_{1}>0$, & Unstable Node \\
\cline { 2 - 3 }$\left(0, \frac{-B_{0}-\sqrt{C_{1}}}{2 A_{0}}\right)$ & $C_{1}=0$ & Saddle \\
\hline \hline \multirow{2}{*}{$(0,0) \in U_{2}$} & $A_{0}<0, C_{1}>0$ & Saddle-Node \\
\hline \hline \multirow{3}{*}{$\left(0, C_{1}>0\right.$} & Stable Node \\
\cline { 2 - 3 } & $A_{0}<0$ & Unstable Node \\
\cline { 2 - 3 } & $0<A_{0}<1$ & Saddle \\
\cline { 2 - 3 } & $A_{0}>1$ & Stable Node \\
\cline { 2 - 3 } & $A_{0}=1, S=1$ & Stable Nodes \\
\hline \hline \multirow{2}{*}{$\left( \pm \sqrt{\left.\frac{1-A_{0}}{S}, 0\right) \in U_{2}}\right)$} & $S=1, S=-1$ & Saddle \\
\cline { 2 - 3 } & $S=-1, A_{0}>1$ & Stable Nodes \\
\hline
\end{tabular}

TABLE 1. Behavior of the singular points for family (K1a). Here $C_{1}=B_{0}^{2}+4 A_{0} S$.

According to the above mentioned when $S=1$ we have the following behaviours: there are three cases for $A_{0}<1$; if $C_{1}<0$ then we obtain the phase portrait (10), if $C_{1}=0$ we have the phase portrait (9) and if $C_{1}>0$ the phase portrait is (8). For $0<A_{0}<1$ the phase portrait is (11) and for $A_{0} \geq 1$ we have (12).

When $S=-1$ the behaviours are as follow: there are three cases for $A_{0}>1$; if $C_{1}<0$ then the phase portrait is (7), if $C_{1}=0$ we have the phase portrait (6) and if $C_{1}>0$ the phase portrait is (5). For $0<A_{0} \leq 1$ there are also three cases: if $C_{1}<0$ the phase portrait is (4), if $C_{1}=0$ then we have the phase portrait (3) and if $C_{1}>0$ the phase portrait is (2). If $A_{0}<1$ we have the phase portrait (1). Finally, if $A_{0}=1$ and $B_{0} \leq 2$ we have again the phase portrait (3). The phase portraits are given in Figure 1. 
Proof of Theorem 4(K1b). We deal here with the differential system

$$
\dot{x}=x y, \quad \dot{y}=S(x-r)(x-1)+A_{0} y^{2},
$$

where $S \in\{-1,1\}, A_{0} \neq 0$ and $r \neq 1$.

System (11) has the following finite singular points: $(1,0),(r, 0)$, and $\left(0, \pm \sqrt{-S r / A_{0}}\right)$. The eigenvalues of the point $(1,0)$ are $\pm \sqrt{S(1-r)}$. Thus, for either $S=1$ and $r<1$ or $S=-1$ and $r>1$ the point is a saddle. When either $S=1$ and $r>1$ or $S=-1$ and $r<1$ we apply Theorem 7 . We first write system (11) in the form given by the Theorem, for that we move the point to the origin, after that we apply the change of variables and time $(x, y, t) \rightarrow(x,-y / \sqrt{S(r-1)}, t / \sqrt{S(r-1)})$ and system (11) becomes

$$
\dot{x}=-y-x y, \quad \dot{y}=x-\frac{x^{2}}{(r-1)}-A_{0} y^{2} .
$$

Then we have $\omega_{1}=\omega_{2}=\omega_{3}=0$, so we have a center.

For the point $(r, 0)$ the eigenvalues are $\pm \sqrt{-S r(1-r)}$. So, when either $S=1$ and $r \notin(0,1)$ or $S=-1$ and $r \in(0,1)$ the point is a saddle. When either $S=1$ and $r \in(0,1)$ or $S=-1$ and $r \notin(0,1)$ we apply again Theorem 7 . By moving the point to the origin and applying the change of variables and time $(x, y, t) \mapsto(x, y \sqrt{r / S(1-r)},-t / \sqrt{S r(1-r)})$ system (11) becomes

$$
\dot{x}=-y-\frac{1}{r} x y, \quad \dot{y}=x+\frac{x^{2}}{r-1}-\frac{A_{0}}{r} y^{2} .
$$

It is easy to see that $\omega_{1}=\omega_{2}=\omega_{3}=0$, so the point is a center.

Finally, if $r=0$ the point becomes $(0,0)$ and is semi-hyperbolic. Indeed, system (11) for $r=0$ is written in the form given in Proposition 5 , so $y=g(x)=\left(A_{0} / S\right) x^{2}+\ldots$, thus we obtain $f(x)=\left(-A_{0} / S^{2}\right) x^{3}+\ldots$, so if $A_{0}>0$ the point is a saddle and if $A_{0}<0$ is a node. The stability of the node is given by $S$ : if $S=1$ it is a stable node and if $S=-1$ it is an unstable node.

The last two points exist only if $A_{0} r S<0$. The point $\left(0, \sqrt{-S r / A_{0}}\right)$ has eigenvalues $\sqrt{-S r / A_{0}}$ and $2 A_{0} \sqrt{-S r / A_{0}}$. Thus, when $A_{0}<0$ we have a saddle and when $A_{0}>0$ we have an unstable node. Similarly, the point $\left(0,-\sqrt{-S r / A_{0}}\right)$ has eigenvalues $-\sqrt{-S r / A_{0}}$ and $-2 A_{0} \sqrt{-S r / A_{0}}$. Thus, when $A_{0}<0$ we have a saddle and when $A_{0}>0$ we have a stable node.

According to the expression (7), system (11) at infinity can be studied from the system $\dot{u}=-u\left(S u^{2}+S r v^{2}-S(r+1) u v+A_{0}-1\right), \quad \dot{v}=-v\left(S u^{2}+S r v^{2}-S(r+1) u v+A_{0}\right)$.

We do not need to study the expression (6) because all the infinite singular points can be studied from the above differential system. We have three infinite singular points: $(0,0)$ and two more points at $\left( \pm \sqrt{\left(1-A_{0}\right) / S}, 0\right)$.

The origin on $U_{2}$ has eigenvalues $-A_{0}, 1-A_{0}$ thus we have an unstable node for $A_{0}<0$; a saddle point for $0<A_{0}<1$ and a stable node for $A_{0}>1$. When $A_{0}=1$ the point is semi-hyperbolic. With a change of time, the above system for $A_{0}=1$ is written in the form given in Proposition 5 and we obtain $f(u)=S u^{3}+\ldots$, so if $S=1$ the point is a stable node and if $S=-1$ a saddle point.

The last two points exist only if $S\left(1-A_{0}\right)>0$. Concerning their stability, the eigenvalues are $-1,-2\left(1-A_{0}\right)$ so if $S=1$ and $A_{0}<1$ the points are stable nodes and if $S=-1$ and $A_{0}>1$ are saddles.

Table 2 summarizes the different possibilities of behavior of the singular points of system 11 depending on the values of the parameters. 


\begin{tabular}{|c|c|c|}
\hline Singular point & Conditions & Type of point \\
\hline \multirow{2}{*}{$(1,0)$} & $S(1-r)>0$ & Saddle \\
\hline & $S(1-r)<0$ & Center \\
\hline \multirow{5}{*}{$(r, 0)$} & $S r(r-1)>0$ & Saddle \\
\hline & $S r(r-1)<0$ & Center \\
\hline & $S=1, r=0, A_{0}<0$ & Stable Node \\
\hline & $S=-1, r=0, A_{0}<0$ & Unstable Node \\
\hline & $r=0, A_{0}>0$ & Saddle \\
\hline \multirow{2}{*}{$\left(0, \pm \sqrt{\frac{-S r}{A_{0}}}\right)$} & $A_{0}<0, S r>0$ & Saddles \\
\hline & $A_{0}>0, S r<0$ & Nodes \\
\hline \multirow{5}{*}{$(0,0) \in U_{2}$} & $A_{0}<0$ & Unstable Node \\
\hline & $0<A_{0}<1$ & Saddle \\
\hline & $A_{0}>1$ & Stable Node \\
\hline & $S=1, A_{0}=1$ & Stable Node \\
\hline & $S=-1, A_{0}=1$ & Saddle \\
\hline \multirow{2}{*}{$\left( \pm \sqrt{\frac{1-A_{0}}{S}}, 0\right) \in U_{2}$} & $S=1, A_{0}<1$ & Stable Nodes \\
\hline & $S=-1, A_{0}>1$ & Saddles \\
\hline
\end{tabular}

TABLE 2. Behavior of the singular points for the family (K1b).

The rest of the cases follow in a similar way as the previous ones. We shall provide the table with the behavior of the singular points and additional important information if needed.

Proof of Theorem $4(K 1 c)$. We deal now with the differential system

$$
\dot{x}=x y, \quad \dot{y}=S\left(x^{2}-1\right)+B_{1} x y+A_{0} y^{2},
$$

where $S^{2}=1, A_{0} \neq 0$ and $B_{1} \geq 0$. Table 3 shows the behavior of all singular points depending on the parameters.

In the case $S=-1, B_{1}=0, A_{0}>0$ we have only two finite singular points and they are centres, this can be proved by applying Theorem 7 . The phase portrait is determined by the value of $A_{0}$. If $0<A_{0} \leq 1$ the phase portrait is (13) and if $A_{0}>1$ the phase portrait is (18) of Figure 1.

The behavior of the all critical points at infinity of system (12) can be studied on $U_{2}$ through the system

$$
\dot{u}=-u\left(S u^{2}-S v^{2}+B_{1} u+A_{0}-1\right), \quad \dot{v}=-v\left(S u^{2}-S v^{2}+B_{1} u+A_{0}\right) .
$$

Proof of Theorem 4(K2a). We deal now with the differential system

$$
\dot{x}=x y, \quad \dot{y}=S x^{2}+y+A_{0} y^{2},
$$

where $S^{2}=1, A_{0} \neq 0$. Table 4 shows the behavior of all singular points depending on the parameters.

According to the expression (7), system (13) at infinity can be studied from the system

$$
\dot{u}=-u\left(S u^{2}+v+A_{0}-1\right), \quad \dot{v}=-v\left(S u^{2}+v+A_{0}\right) .
$$

We do not need to study the expression (6) because all the infinite singular points can be studied from the above differential system. 


\begin{tabular}{|c|c|c|}
\hline Singular point & Conditions & Type of point \\
\hline \multirow{4}{*}{$(1,0)$} & $S=1$ & Saddle \\
\hline & $S=-1, B_{1} \geq 2 \sqrt{2}$ & Unstable Node \\
\hline & $S=-1,0<B_{1}<2 \sqrt{2}$ & Unstable Focus \\
\hline & $S=-1, B_{1}=0$ & Center \\
\hline \multirow{4}{*}{$(-1,0)$} & $S=1$ & Saddle \\
\hline & $S=-1, B_{1} \geq 2 \sqrt{2}$ & Stable Node \\
\hline & $S=-1,0<B_{1}<2 \sqrt{2}$ & Stable Focus \\
\hline & $S=-1, B_{1}=0$ & Center \\
\hline \multirow{2}{*}{$\left(0, \pm \sqrt{\frac{S}{A_{0}}}\right)$} & $S=1, A_{0}>0$ & Nodes \\
\hline & $S=-1, A_{0}<0$ & Saddles \\
\hline \multirow{4}{*}{$(0,0) \in U_{2}$} & $A_{0}<0$ & "Unstable Node \\
\hline & $0<A_{0}<1$ & Saddle \\
\hline & $A_{0}>1$ & Stable Node \\
\hline & $A_{0}=1$ & Saddle-Node \\
\hline \multirow{4}{*}{$\left(\frac{-B_{1}+\sqrt{C_{2}}}{2 S}, 0\right) \in U_{2}$} & $C_{2}>0, A_{0}<1$ & Stable Node \\
\hline & $C_{2}>0, A_{0}>1$ & Saddle \\
\hline & $A_{0}=1$ & Saddle-Node \\
\hline & $C_{2}=0$ & Saddle-Node \\
\hline \multirow{5}{*}{$\left(\frac{-B_{1}-\sqrt{C_{2}}}{2 S}, 0\right) \in U_{2}$} & $C_{2}>0, S=1$ & Stable Node \\
\hline & $C_{2}>0, S=-1$ & Saddle \\
\hline & $A_{0}=1, S=-1$ & Saddle \\
\hline & $A_{0}=1, S=1$ & Stable Node \\
\hline & $C_{2}=0$ & Saddle-Node \\
\hline
\end{tabular}

TABLE 3. Behavior of the singular points for the family (K1c). Here $C_{2}=B_{1}^{2}-$ $4 A_{0} S+4 S$.

Proof of Theorem 4(K2b). We deal now with the differential system

$$
\dot{x}=x y, \quad \dot{y}=S(x-r)^{2}+y+A_{0} y^{2},
$$

where $S^{2}=1, A_{0} \neq 0, r \in\{0,1\}$. In Table 5 we summarize the behavior of all singular points depending on the parameters.

We consider two cases: $r=1$ and $r=0$. For the case $r=1$ system (14) has three finite singular point: $(1,0)$ and $\left(0, \pm \sqrt{\left.-S / A_{0}\right)}\right)$. The point $(1,0)$ is semi-hyperbolic. By applying Proposition 6 we have $y=f(x)=0$ and $G(x, f(x))=S x^{2}+\ldots$, moreover $\Phi=0$ then the point is a cusp. The phase portraits corresponding to the cusp are (41), (42), (45) and (46), depending on the value of $A_{0}$ and $S$.

The infinite singular points of system (14) with $r=1$ can be studied from the system

$$
\dot{u}=-u\left(S u^{2}-2 S u v+S v^{2}+A_{0}-1\right), \quad \dot{v}=-v\left(S u^{2}-2 S u v+S v^{2}+A_{0}\right),
$$

on $U_{2}$, and we do not need to study the expression (6) because all the infinite singular points can be studied from the above differential system. 


\begin{tabular}{|c|c|c|}
\hline Singular point & Conditions & Type of point \\
\hline \hline \multirow{2}{*}{$(0,0)$} & $S=1$ & Saddle \\
\cline { 2 - 3 }$\left(0,-\frac{1}{A_{0}}\right)$ & $S=-1$ & Unstable Node \\
\hline \hline \multirow{2}{*}{$(0,0) \in U_{2}$} & $A_{0}>0$ & Stable Node \\
\hline \hline \multirow{2}{*}{$\left(A_{0}<0\right.$} & Saddle \\
\cline { 2 - 3 } & $0<A_{0}<1$ & Snstable Node \\
\cline { 2 - 3 } & $A_{0}>1$ & Stable Node \\
\cline { 2 - 3 } & $A_{0}=1, S=1$ & Stable Node \\
\cline { 2 - 3 } & $A_{0}=1, S=-1$ & Saddle \\
\hline \hline$\left( \pm \sqrt{\frac{1-A_{0}}{S}}, 0\right) \in U_{2}$ & $S=1, A_{0}<1$ & Stable Nodes \\
\cline { 2 - 3 } & $S=-1, A_{0}>1$ & Saddles \\
\hline
\end{tabular}

TABLE 4. Behavior of the singular points for the family (K2a).

\begin{tabular}{|c|c|c|}
\hline Singular point & Conditions & Type of point \\
\hline \hline \multirow{2}{*}{$(r, 0)$} & $r=0$ & Degenerate \\
\cline { 2 - 3 }$\left(0, \pm \sqrt{-\frac{S}{A_{0}}}\right)$ & $r=1, A_{0}>0, S=1$ & Cusp \\
\cline { 2 - 3 } & $r=1, A_{0}<0, S=-1$ & Saddles \\
\hline \hline \multirow{2}{*}{$(0,0) \in U_{2}$} & $A_{0}<0$ & Unstable Node \\
\cline { 2 - 3 } & $0<A_{0}<1$ & Saddle \\
\cline { 2 - 3 } & $A_{0}>1$ & Stable Node \\
\cline { 2 - 3 } & $A_{0}=1, S=1$ & Stable Node \\
\hline \hline \multirow{2}{*}{$\left( \pm \sqrt{\frac{1-A_{0}}{S}}, 0\right) \in U_{2}=1, S=-1$} & Saddle \\
\cline { 2 - 3 } & $S=1, A_{0}<1$ & Stable Nodes \\
\hline
\end{tabular}

TABLE 5. Behavior of the singular points for the family (K2b).

For the case $r=0$ system (14) has only one finite singular point: $(0,0)$. This point is degenerate so we determined its behavior by applying the blow-up technique (for more details see [2]). Starting from system (14) with $r=0$ we apply the change $(x, y) \rightarrow(x, p y)$, where $p$ is a new variable, to obtain (after cancelling a common factor $y$ )

$$
\dot{p}=-p\left(S p^{2}+A_{0}-1\right), \quad \dot{y}=y\left(S p^{2}+A_{0}\right) .
$$

This new system has three singular points on $y=0:(0,0),\left( \pm \sqrt{\left(1-A_{0}\right) / S}, 0\right)$. The eigenvalues of $(0,0)$ are $A_{0}$ and $1-A_{0}$ so either if $A_{0}<0$ or $A_{0}>1$ then the point is a saddle, if $0<A_{0}<1$ then the point is an unstable node and if $A_{0}=1$ the point is semihyperbolic. Applying proposition (5) we prove that the point is unstable node if $S=-1$ and a saddle if $S=1$. 
On the other hand, the points $\left( \pm \sqrt{\left(1-A_{0}\right) / S}, 0\right)$ have eigenvalues $1,-2\left(1-A_{0}\right)$ so they are saddles if $A_{0}<1$ and unstable nodes if $A_{0}>1$. Finally, undoing the blow-up we obtain the local behaviour of the point $(0,0)$ of system $(14)$ with $r=0$.

According to the expression (7) the infinite singular points of system (14) for the case $r=0$ can be studied from the system

$$
\dot{u}=-u\left(S u^{2}+A_{0}-1\right), \quad \dot{v}=-v\left(S u^{2}+A_{0}\right),
$$

on $U_{2}$. Since in the above system we can study all the infinite singular points we do not need to study the expression (6).

Proof of Theorem $4(K 2 c)$. We deal now with the differential system

$$
\dot{x}=x y, \quad \dot{y}=S x^{2}+B_{1} x y+A_{0} y^{2},
$$

where $S^{2}=1, A_{0} \neq 0$ and $B_{1}>0$. Table 6 shows the behavior of all singular points depending on the parameters.

\begin{tabular}{|c|c|c|}
\hline Singular point & Conditions & Type of point \\
\hline \hline \multirow{3}{*}{$(0,0)$} & & Degenerate \\
\hline \hline \multirow{4}{*}{$\left(\frac{-B_{1}+\sqrt{C_{2}}}{2 S}, 0\right) \in U_{2}$} & $A_{0}<0$ & Unstable Node \\
\cline { 2 - 3 } & $0<A_{0}<1$ & Saddle \\
\cline { 2 - 3 } & $A_{0}>1$ & Stable Node \\
\cline { 2 - 3 } & $A_{0}=1$ & Saddle-Node \\
\cline { 2 - 3 } & $C_{2}>0, A_{0}<1$ & Stable Node \\
\cline { 2 - 3 } & $A_{0}=1$ & Saddle \\
\hline \hline \multirow{5}{*}{$\left(\frac{-B_{1}-\sqrt{C_{2}}}{2 S}, 0\right) \in U_{2}$} & $C_{2}=0$ & Saddle-Node \\
\cline { 2 - 3 } & $C_{2}>0, S=1$ & Stable Node \\
\cline { 2 - 3 } & $C_{2}>0, S=-1$ & Saddle \\
\cline { 2 - 3 } & $A_{0}=1, S=-1$ & Saddle \\
\cline { 2 - 3 } & $A_{0}=1, S=1$ & Stable Node \\
\cline { 2 - 3 } & $C_{2}=0$ & Saddle-Node \\
\hline
\end{tabular}

TABLE 6. Behavior of the singular points for the family (K2c). Here again $C_{2}=$ $B_{1}^{2}-4 A_{0} S+4 S$.

System (15) has only one finite singular point: $(0,0)$. This point is degenerate so, again as in case $(K 2 b)$, we use the blow-up technique to known its behavior.

System (15) at infinity can be studied from the system

$$
\dot{u}=-u\left(S u^{2}+B_{1} u+A_{0}-1\right), \quad \dot{v}=-v\left(S u^{2}+B_{1} u+A_{0}\right) .
$$

This system has three singular points: $(0,0)$ and two more points at $\left(\left(-B_{1} \pm \sqrt{C_{2}}\right) / 2 S, 0\right)$ where $C_{2}:=B_{1}^{2}-4 A_{0} S+4 S$. These points only exist if $C_{2} \geq 0$. When $C_{2}=0$ the point $\left(-B_{1} / 2 S, 0\right)$ is a semi-hyperbolic singular point. Applying Proposition 5 we have $v=f(u)=0$ so $G(u, f(u))=-\left(B_{1} / 2\right) u^{2}+\ldots$ thus the point is a saddle-node. When $A_{0}=1$ the point $(0,0)$ on $U_{2}$ is a semi-hyperbolic singular point. By using Proposition 5 we prove that the point is a saddle-node.

Proof of Theorem 4(K3a). We deal now with the differential system

$$
\dot{x}=x y, \quad \dot{y}=S\left(x^{2}+1\right)+B_{0} y+A_{0} y^{2},
$$


where $S^{2}=1, A_{0} \neq 0$ and $B_{0}>0$. Table 7 shows the behavior of all singular points depending on the parameters.

\begin{tabular}{|c|c|c|}
\hline Singular point & Conditions & Type of point \\
\hline \hline \multirow{2}{*}{$\left(0,-\frac{B_{0}+\sqrt{C_{3}}}{2 A_{0}}\right)$} & $C_{3}>0, S=1$ & Saddle \\
\cline { 2 - 3 }$\left(0,-\frac{B_{0}-\sqrt{C_{3}}}{2 A_{0}}\right)$ & $C_{3}>0, S=-1$ & Unstable Node \\
\hline \hline \multirow{3}{*}{$(0,0) \in U_{2}$} & $A_{0}<0, C_{3}>0$ & Saddle \\
\cline { 2 - 3 } & $A_{0}>0, C_{3}>0$ & Stable Node \\
\cline { 2 - 3 } & $C_{3}=0$ & Saddle-Node \\
\hline \hline \multirow{3}{*}{$\left(A_{0}<0\right.$} & Unstable Node \\
\cline { 2 - 3 } & $0<A_{0}<1$ & Saddle \\
\cline { 2 - 3 } & $A_{0}>1$ & Stable Node \\
\cline { 2 - 3 } & $A_{0}=1, S=1$ & Stable Node \\
\hline \hline \multirow{2}{*}{$\left( \pm \sqrt{\frac{1-A_{0}}{S}}, 0\right) \in U_{2}$} & $A_{0}=1, S=-1$ & Saddle \\
\cline { 2 - 3 } & $A_{0}<1$ & Stable Nodes \\
\hline
\end{tabular}

TABLE 7. Behavior of the singular points for the family (K3a). Here $C_{3}:=B_{0}^{2}-4 A_{0} S$.

System (16) has two singular points $\left(0,\left(-B_{0} \pm \sqrt{B_{0}^{2}-4 A_{0} S}\right) / 2 A_{0}\right)$. Let $C_{3}:=B_{0}^{2}-4 A_{0} S$ be so the points only exist if $C_{3} \geq 0$. For the case $C_{3}=0$ the critical point becomes $\left(0,-2 S / B_{0}\right)$ with eigenvalues 0 and $-2 S / B_{0}$ so is a semi-hyperbolic singular point. Applying Proposition 5 we have $y=f(x)=0$ so $G(x, f(x))=-\left(B_{0}^{3} / 8 S^{2}\right) x^{2}+\ldots$, thus the point is a saddle-node. The phase portrait in this case corresponds to (49) and (52) of Figure 1 depending of the value of $A_{0}$.

The behavior of system (16) at infinity can be studied from the system

$$
\dot{u}=-u\left(S v^{2}+S u^{2}+B_{0} v+A_{0}-1\right), \quad \dot{v}=-v\left(S v^{2}+S u^{2}+B_{0} v+A_{0}\right) .
$$

Proof of Theorem 4(K3b). We deal now with the differential system

$$
\dot{x}=x y, \quad \dot{y}=S\left((x-a)^{2}+1\right)+A_{0} y^{2},
$$

where $S^{2}=1, A_{0} \neq 0$ and $a \in \mathbb{R}$. Table 8 shows the behavior of all singular points depending on the parameters.

According to the expression (7), system (16) at infinity can be studied from the system

$$
\dot{u}=-u\left(S(a v-u)^{2}+S v^{2}+A_{0}-1\right), \quad \dot{v}=-v\left(S(a v-u)^{2}+S v^{2}+A_{0}\right) .
$$

The behavior of the infinite singular points is the same as in the case (K3a).

Proof of Theorem 4(K3c). We deal now with the differential system

$$
\dot{x}=x y, \quad \dot{y}=S\left(x^{2}+1\right)+B_{1} x y+A_{0} y^{2},
$$

where $S^{2}=1, A_{0} \neq 0$ and $B_{1}>0$. Table 9 shows the behavior of all singular points depending on the parameters.

The behavior of system (18) at infinity can be studied from the system

$$
\dot{u}=-u\left(S u^{2}+S v^{2}+B_{1} u+A_{0}-1\right), \quad \dot{v}=-v\left(S u^{2}+S v^{2}+B_{1} u+A_{0}\right) .
$$

The above system has at most three singular points: $(0,0)$ and $\left(\left(-B_{1} \pm \sqrt{C_{2}}\right) / 2 S, 0\right)$ where $C_{2}:=B_{1}^{2}-4 A_{0} S+4 S$. If $A_{0}=1$ the point $(0,0)$ is semi-hyperbolic and applying Proposition 5 it can be proved that it is a saddle-node. For the case $C_{2}=0$, again by using 


\begin{tabular}{|c|c|c|}
\hline Singular point & Conditions & Type of point \\
\hline \hline$\left(0, \sqrt{\frac{-S\left(a^{2}+1\right)}{A_{0}}}\right)$ & $A_{0}<0, S=1$ & Saddle \\
\cline { 2 - 3 } & $A_{0}>0, S=-1$ & Unstable Node \\
\hline \hline$\left(0,-\sqrt{\frac{-S\left(a^{2}+1\right)}{A_{0}}}\right)$ & $A_{0}<0, S=1$ & Saddle \\
\cline { 2 - 3 } & $A_{0}>0, S=-1$ & Stable Node \\
\hline \hline \multirow{3}{*}{$(0,0) \in U_{2}$} & $A_{0}<0$ & Unstable Node \\
\cline { 2 - 3 } & $0<A_{0}<1$ & Saddle \\
\cline { 2 - 3 } & $A_{0}>1$ & Stable Node \\
\cline { 2 - 3 } & $A_{0}=1, S=1$ & Stable Node \\
\cline { 2 - 3 } & $A_{0}=1, S=-1$ & Saddle \\
\hline \hline \multirow{2}{*}{$\left( \pm \sqrt{\frac{1-A_{0}}{S}}, 0\right) \in U_{2}$} & $A_{0}<1$ & Stable Nodes \\
\cline { 2 - 3 } & $A_{0}>1$ & Saddles \\
\hline
\end{tabular}

TABLE 8. Behavior of the singular points for the family (K3b).

Proposition 5, we prove that the point $\left(-B_{1} / 2 S, 0\right)$ is a saddle-node. The phase portrait in this case corresponds to (53) or (56) of Figure 1 depending of the value of $A_{0}$.

\begin{tabular}{|c|c|c|}
\hline Singular point & Conditions & Type of point \\
\hline \hline \multirow{2}{*}{$\left(0, \sqrt{-\frac{S}{A_{0}}}\right)$} & $S=1, A_{0}<0$ & Saddle \\
\cline { 2 - 3 }$\left(0,-\sqrt{-\frac{S}{A_{0}}}\right)$ & $S=-1, A_{0}>0$ & Unstable Node \\
\cline { 2 - 3 } & $S=-1, A_{0}<0$ & Saddle \\
\hline \hline \multirow{5}{*}{$(0,0) \in U_{2}>0$} & Stable Node \\
\hline \multirow{5}{*}{$\left(\frac{-B_{1}+\sqrt{C_{2}}}{2 S}, 0\right) \in U_{2}$} & $A_{0}<0$ & Unstable Node \\
\cline { 2 - 3 } & $C_{2}>0, A_{0}<1$ & Saddle \\
\cline { 2 - 3 } & $A_{0}>1$ & Stable Node \\
\cline { 2 - 3 } & $A_{0}=1$ & Saddle-Node \\
\cline { 2 - 3 } & $C_{2}>0, A_{0}<1$ & Stable Node \\
\hline \hline \multirow{5}{*}{$\left(\frac{-B_{1}-\sqrt{C_{2}}}{2 S}, 0\right) \in U_{2}=0$} & Saddle \\
\cline { 2 - 3 } & $C_{2}>0, S=1$ & Saddle-Node \\
\cline { 2 - 3 } & $C_{2}>0, S=-1$ & Stable Node \\
\cline { 2 - 3 } & $A_{0}=1, S=-1$ & Saddle \\
\hline & $A_{0}=1, S=1$ & Saddle \\
\cline { 2 - 3 } & $C_{2}=0$ & Stable Node \\
\hline
\end{tabular}

TABLE 9 . Behavior of the singular points for the family (K3c). Here $C_{2}=B_{1}^{2}-$ $4 A_{0} S+4 S$. 
Proof of Theorem $4(K 4 a)$. We deal now with the differential system

$$
\dot{x}=x y, \quad \dot{y}=-T+x+A_{0} y^{2},
$$

where $A_{0} \neq 0$ and $T \in\{-1,0,1\}$. Table 10 shows the behavior of all singular points depending on the parameters.

System (19) has at most three finite singular points: $(T, 0)$ and $\left(0, \pm \sqrt{T / A_{0}}\right)$. For the case of the point $(T, 0)$ the eigenvalues are $\sqrt{T}$ and $-\sqrt{T}$, so the point is a saddle if $T=1$ and it is a center if $T=-1$. It can be proved by moving the singular point to the origin and applying Theorem 7 to the system obtained: $\dot{x}=-y+x y, \dot{y}=x+A_{0} y^{2}$, the phase portraits in this case are (59)-(62) of Figure 1 depending of the value of $A_{0}$.

If $T=0$ then according to Proposition 6 the point is a saddle if $A_{0}>0$ and is an unstable node if $A_{0}<0$.

Following the expression (6) and (7), the study of the behavior of the singular points at infinity can be done through the systems

$$
\begin{gathered}
\dot{u}=\left(A_{0}-1\right) u^{2}+v-T v^{2}, \quad \dot{v}=-u v, \\
\dot{u}=-u\left(-T v^{2}+u v+A_{0}-1\right), \quad \dot{v}=-v\left(-T v^{2}+u v+A_{0}\right),
\end{gathered}
$$

on $U_{1}$ and $U_{2}$ respectively. Note that when $A_{0}=1$ the infinity is degenerate. System (20) has a singular point: $(0,0)$, which is semi-hyperbolic. Applying Proposition 5 we have $v=g(u)=\left(1-A_{0}\right)+\ldots$ and then $F(u, g(u))=\left(A_{0}-1\right) u^{3}+\ldots$ So if $A_{0}>1$ the point is an unstable node and if $A_{0}<1$ is a saddle.

\begin{tabular}{|c|c|c|}
\hline Singular point & Conditions & Type of point \\
\hline \hline \multirow{4}{*}{$(T, 0)$} & $T=-1$ & Center \\
\cline { 2 - 3 } & $T=1$ & Saddle \\
\cline { 2 - 3 } & $T=0, A_{0}>0$ & Saddle \\
\cline { 2 - 3 } & $T=0, A_{0}<0$ & Unstable Node \\
\hline \hline \multirow{2}{*}{$\left(0, \pm \sqrt{\frac{T}{A_{0}}}\right)$} & $T=1, A_{0}>0$ & Nodes \\
\cline { 2 - 3 } & $T=-1, A_{0}<0$ & Saddles \\
\hline \hline \multirow{2}{*}{$(0,0) \in U_{1}$} & $A_{0}>1$ & Unstable Node \\
\cline { 2 - 3 } & $A_{0}<1$ & Saddle \\
\hline \hline \multirow{2}{*}{$(0,0) \in U_{2}$} & $A_{0}<0$ & Unstable Node \\
\cline { 2 - 3 } & $0<A_{0}<1$ & Saddle \\
\cline { 2 - 3 } & $A_{0}>1$ & Stable Node \\
\hline
\end{tabular}

TABLE 10. Behavior of the singular points for the family (K4a). If $A_{0}=1$ then the infinity is degenerate.

Proof of Theorem 4(K5a). We deal now with the differential system

$$
\dot{x}=x y, \quad \dot{y}=S+B_{0} y+A_{0} y^{2},
$$

where $S^{2}=1, A_{0} \neq 0$ and $B_{0}>0$. The behavior of all singular points depending on the parameters is shown in Table 11 .

System $(22)$ has at most two finite singular point: $\left(0,\left(-B_{0} \pm \sqrt{B_{0}^{2}-4 A_{0} S}\right) / 2 A_{0}\right)$. The existence of the points depend on the value of the discriminant $C_{3}:=B_{0}^{2}-4 A_{0} S$. When $C_{3}=0$ by applying Proposition 5 we prove that the point $\left(0,-2 S / B_{0}\right)$ is a saddle-node and depending of the value of $A_{0}$ we have the phase portrait (72), (80) or (81), given in Figure 1. 
Following (6) and (7), system (22) at infinity can be studied from the systems

$$
\begin{gathered}
\dot{u}=S v^{2}+B_{0} u v+\left(A_{0}-1\right) u^{2}, \quad \dot{v}=-u v, \\
\dot{u}=-u\left(S v^{2}+B_{0} v+A_{0}-1\right), \quad \dot{v}=-v\left(S v^{2}+B_{0} v+A_{0}\right),
\end{gathered}
$$

on $U_{1}$ and $U_{2}$ respectively. Note that if $A=1$ the infinity is degenerate.

On the other hand, for $A_{0} \neq 1$ system (23) has a singular point: $(0,0)$, such a point is degenerate. To obtain its local behavior we apply the blow-up technique.

\begin{tabular}{|c|c|c|}
\hline Singular point & Conditions & Type of point \\
\hline \hline \multirow{2}{*}{$\left(0,-\frac{B_{0}+\sqrt{C_{3}}}{2 A_{0}}\right)$} & $C_{3}>0, S=1$ & Saddle \\
\cline { 2 - 3 } & $C_{3}>0, S=-1$ & Unstable Node \\
\hline \hline \multirow{3}{*}{$\left(0,-\frac{B_{0}-\sqrt{C_{3}}}{2 A_{0}}\right)$} & $A_{0}<0, C_{3}>0$ & Saddle \\
\cline { 2 - 3 } & $A_{0}>0, C_{3}>0$ & Stable Node \\
\cline { 2 - 3 } & $C_{3}=0$ & Saddle-Node \\
\hline \hline$(0,0) \in U_{1}$ & $A_{0} \neq 1$ & Degenerate \\
\hline \hline \multirow{2}{*}{$(0,0) \in U_{2}$} & $A_{0}<0$ & Unstable Node \\
\cline { 2 - 3 } & $0<A_{0}<1$ & Saddle \\
\cline { 2 - 3 } & $A_{0}>1$ & Stable Node \\
\hline
\end{tabular}

TABLE 11. Behavior of the singular points for the family (K5a). Here again $C_{3}:=B_{0}^{2}-4 A_{0} S$. If $A_{0}=1$ then the infinity is degenerate.

Proof of Theorem 4(K5b). We deal now with the differential system

$$
\dot{x}=x y, \quad \dot{y}=S+A_{0} y^{2},
$$

where $S^{2}=1, A_{0} \neq 0$. In Table 12 we give the behavior of the singular points depending on the parameters.

According to (6) and (7), system (25) at infinity can be studied from the systems

$$
\begin{gathered}
\dot{u}=\left(A_{0}-1\right) u^{2}+S v^{2}, \quad \dot{v}=-u v, \\
\dot{u}=-u\left(S v^{2}+A_{0}-1\right), \quad \dot{v}=-v\left(S v^{2}+A_{0}\right) .
\end{gathered}
$$

The behavior at infinity is the same as in case (K5a).

\begin{tabular}{|c|c|c|}
\hline Singular point & Conditions & Type of point \\
\hline \hline \multirow{2}{*}{$\left(0, \sqrt{-\frac{S}{A_{0}}}\right)$} & $S=1, A_{0}<0$ & Saddle \\
\cline { 2 - 3 } & $S=-1, A_{0}>0$ & Unstable Node \\
\hline \hline \multirow{2}{*}{$\left(0,-\sqrt{-\frac{S}{A_{0}}}\right)$} & $S=1, A_{0}<0$ & Saddle \\
\cline { 2 - 3 } & $S=-1, A_{0}>0$ & Stable Node \\
\hline \hline$(0,0) \in U_{1}$ & $A_{0} \neq 1$ & Degenerate \\
\hline \hline \multirow{2}{*}{$(0,0) \in U_{2}$} & $A_{0}<0$ & Unstable Node \\
\cline { 2 - 3 } & $0<A_{0}<1$ & Saddle \\
\cline { 2 - 3 } & $A_{0}>1$ & Stable Node \\
\hline
\end{tabular}

TABLE 12. Behavior of the singular points for the family (K5b). 
Proof of Theorem 4(K5c). We deal now with the differential system

$$
\dot{x}=x y, \quad \dot{y}=S+x y+A_{0} y^{2},
$$

where $S^{2}=1, A_{0} \neq 0$. The behavior of all singular points depending on the parameters are shown in Table 13.

As stated by expressions (6) and (7), system (28) at infinity can be studied from systems

$$
\begin{gathered}
\dot{u}=\left(A_{0}-1\right) u^{2}+S v^{2}+u, \quad \dot{v}=-u v, \\
\dot{u}=-u\left(S v^{2}+u+A_{0}-1\right), \quad \dot{v}=-v\left(S v^{2}+u+A_{0}\right),
\end{gathered}
$$

on $U_{1}$ and $U_{2}$ respectively. System (30) has two singular points: $(0,0)$ and $\left(1-A_{0}, 0\right)$. When $A_{0}=1$ the point is semi-hyperbolic. Doing a change of time to system (30) and applying Proposition 5 we prove that the point is a saddle-node. So the phase portraits given in Figure 1 that correspond to this case are (53) if $S=-1$ or (57) if $S=1$.

With respect to system (29), the origin is the only singular point and it is semi-hyperbolic. Applying Proposition 5 we have $v=g(u)=-S u^{2}+\ldots$, and hence $F(u, g(u))=f(u)=$ $S u^{3}+\ldots$, so if $S=1$ then the point is an unstable node and if $S=-1$ then it is a saddle.

\begin{tabular}{|c|c|c|}
\hline Singular point & Conditions & Type of point \\
\hline \hline \multirow{2}{*}{$\left(0, \sqrt{-\frac{S}{A_{0}}}\right)$} & $S=1, A_{0}<0$ & Saddle \\
\cline { 2 - 3 } & $S=-1, A_{0}>0$ & Unstable Node \\
\hline \hline \multirow{2}{*}{$\left(0,-\sqrt{-\frac{S}{A_{0}}}\right)$} & $S=1, A_{0}<0$ & Saddle \\
\cline { 2 - 3 } & $S=-1, A_{0}>0$ & Stable Node \\
\hline \hline \multirow{2}{*}{$(0,0) \in U_{1}$} & $S=1$ & Unstable Node \\
\cline { 2 - 3 } & $S=-1$ & Saddle \\
\hline \hline \multirow{3}{*}{$(0,0) \in U_{2}$} & $A_{0}<0$ & Unstable Node \\
\cline { 2 - 3 } & $0<A_{0}<1$ & Saddle \\
\cline { 2 - 3 } & $A_{0}>1$ & Stable Node \\
\cline { 2 - 3 } & $A_{0}=1$ & Saddle-Node \\
\hline
\end{tabular}

TABLE 13. Behavior of the singular points for the family (K5c).

\section{INVARIANT STRAIGHT LINES}

We compute in this last section the invariant straight lines of the differential systems appearing in Proposition 2. When real, they appear in the phase portraits of Figure 1.

We recall that an algebraic curve $f=0, f \in \mathbb{C}[x, y]$, is invariant under the flow of a differential system $\dot{x}=P, \dot{y}=Q$ of degree $m \in \mathbb{N}$ if there exists $k \in \mathbb{C}[x, y]$, $\operatorname{deg} k<m$, such that

$$
P \frac{\partial f}{\partial x}+Q \frac{\partial f}{\partial y}=k f
$$

We call this polynomial $k$ the cofactor of $f=0$.

Table 14 shows the invariant straight lines, that is the invariant algebraic curves $f=0$ of degree 1, of each family of differential systems appearing in Proposition 2, besides $x=0$. Of course $x=0$ is invariant because $x \mid \dot{x}$, and hence equation (31) is satisfied with $k=y$. A family not appearing in this table is a family without invariant straight lines besides $x=0$. 


\begin{tabular}{|c|c|c|}
\hline Family & Conditions & Straight lines \\
\hline \multirow{2}{*}{$(\mathrm{K} 1 \mathrm{a})$} & $B_{0}=\left(1-2 A_{0}\right) \sqrt{S /\left(1-A_{0}\right)}$ & $\sqrt{S}(1 \pm x)-\sqrt{1-A_{0}} y=0$ \\
\hline & $B_{0}=-\left(1-2 A_{0}\right) \sqrt{S /\left(1-A_{0}\right)}$ & $\sqrt{S}(1 \pm x)+\sqrt{1-A_{0}} y=0$ \\
\hline \multirow{2}{*}{$(\mathrm{K} 1 \mathrm{~b})$} & $A_{0}=1 /(1-r)$ & $\sqrt{r-1}(x-r) \pm \sqrt{r S} y=0$ \\
\hline & $A_{0}=r /(r-1)$ & $\sqrt{(1-r) S(x-1) \pm y=0}$ \\
\hline \multirow{2}{*}{$(\mathrm{K} 1 \mathrm{c})$} & $B_{1}=\left(1-2 A_{0}\right) \sqrt{S / A_{0}}$ & $\sqrt{S}(x \pm 1)-\sqrt{A_{0}} y=0$ \\
\hline & $B_{1}=-\left(1-2 A_{0}\right) \sqrt{S / A_{0}}$ & $\sqrt{S}(x \pm 1)+\sqrt{A_{0}} y=0$ \\
\hline$(\mathrm{K} 2 \mathrm{~b})$ & $r=0$ & $\sqrt{S} x \pm \sqrt{1-A_{0}} y=0$ \\
\hline$(\mathrm{K} 2 \mathrm{c})$ & & $\left(B_{1} \pm \sqrt{B_{1}^{2}+4 S\left(1-A_{0}\right)}\right) x+2\left(A_{0}-1\right) y=0$ \\
\hline \multirow{2}{*}{$(\mathrm{K} 3 \mathrm{a})$} & $B_{0}=\left(1-2 A_{0}\right) b \sqrt{S /\left(A_{0}-1\right)}$ & $\sqrt{S}(b i \pm x)-\sqrt{1-A_{0}} y=0$ \\
\hline & $B_{0}=\left(1-2 A_{0}\right) b \sqrt{S /\left(A_{0}-1\right)}$ & $\sqrt{S}(b i \pm x)+\sqrt{1-A_{0}} y=0$ \\
\hline$(\mathrm{K} 3 \mathrm{~b})$ & $A_{0}=1 / 2, a=0$ & $\begin{array}{l}\sqrt{2 S}(x \pm i)+y=0 \\
\sqrt{2 S}(x \pm i)-y=0 \\
\end{array}$ \\
\hline \multirow{2}{*}{$(\mathrm{K} 3 \mathrm{c})$} & $B_{1}=\left(1-2 A_{0}\right) \sqrt{S / A_{0}}$ & $\sqrt{S}(x \pm i)-\sqrt{A_{0}} y=0$ \\
\hline & $B_{1}=-\left(1-2 A_{0}\right) \sqrt{S / A_{0}}$ & $\sqrt{S}(x \pm i)+\sqrt{A_{0}} y=0$ \\
\hline$(\mathrm{K} 4 \mathrm{a})$ & $A_{0}=1, T^{2}=1$ & $x-T \pm \sqrt{T} y=0$ \\
\hline$(\mathrm{K} 5 \mathrm{a})$ & & $\left(B_{0} \pm \sqrt{B_{0}^{2}-4 S A_{0}}\right)+2 A_{0} y=0$ \\
\hline$(\mathrm{K} 5 \mathrm{~b})$ & & $\sqrt{S} \pm i \sqrt{A_{0}} y=0$ \\
\hline
\end{tabular}

TABLE 14. Complex invariant straight lines of each family of differential systems appearing in Proposition 2 besides $x=0$. A family not appearing in this table is a family with only one invariant straight line, $x=0$.

\section{ACKNOWLEDGEMENTS}

A. Ferragut is supported by the Ministerio de Economía, Industria y Competitividad Agencia Estatal de Investigación grant MTM2016-77278-P (FEDER) and by the Universitat Jaume I grant P1-1B2015-16. J. D. García-Saldaña is supported by FONDECyT grant number 11171115/2017. C. Valls is supported by Portuguese National Funds through FCT - Fundação para a Ciência e a Tecnologia within the project PTDC/MAT/117106/2010 and by CAMGSD.

\section{REFERENCES}

[1] G. Alobaidi amd R. Mailler, On the Abel equation of the second kind with sinusoidal forcing, Nonlinear Analysis: Modeling and Control, 12 (2007), 33-44.

[2] M.J. Álvarez, A. Ferragut and X. Jarque A survey on the blow up technique, Int. J. Bifurcation and Chaos 21 (2011), 3103-3118.

[3] A.A. Andronov, E.A. Leontovich, I.I. Gordon and A.G. Maier, Qualitative theory of secondorder dynamic systems, John Wiley and Sons, 1973.

[4] V. I. Arnol'd and Y. S. Ilyashenko, Dynamical Systems I, Ordinary Differential Equations. Encyclopaedia of Mathematical Sciences, Vol. 1-2, Springer-Verlag, Heidelberg, 1988.

[5] L. Cairó and J. Llibre, Phase portraits of quadratic polynomial vector fields having a rational first integral of degree 2, Nonlinear Anal., Ser. A: Theory Methods 67 (2007), 327-348.

[6] J. Chavarriga, H. Giacomini, J. Giné and J. Llibre, On the integrability of two-dimensional flows, J. Differential Equations 157 (1999), 163-182.

[7] A. Ferragut and C. Valls, Phase portraits of Abel quadratic differential systems of second kind, Dyn. Syst. (2017), DOI 10.1080/14689367.2017.1402296.

[8] E.A.V. Gonzales, Generic properties of polynomial vector fields at infinity, Trans. Amer. Math. Soc. 143 (1969), 201-222.

[9] C. Li, Two problems of planar quadratic systems, Scientia Sinica (Series A) 26 (1983), 471-481.

[10] D.A. Neumann, Classification of continuous flows on 2-manifolds, Proc. Amer. Math. Soc. 48 (1975), $73-81$. 
[11] N. Rozov Encyclopeida of Mathematics. Available online at http:///www.encyclopediaofmath.org/index.php?title=Abel_differential_equation(and)oldid=18925.

[12] J. Sotomayor, Liçóes de Equaçóes Diferenciais Ordinárias, IMPA, Rio de Janerio, 1979.

[13] Ye Yanqian et Al., Theory of Limit Cycles, Transl. Math. Monographs 66, Amer. Math. Soc., Providence, 1984.

A. Ferragut: Departament de Matemàtiques and imaC; Universitat Jaume I, Av. de Vicent Sos Baynat, s/n, Campus del Riu Sec, 12071 Castelló de la Plana, Spain

E-mail address: ferragut@uji.es

J.D. García-Saldaña: Departamento de Matemática y Física Aplicadas, Universidad Católica de la Santísima Concepción, Alonso de Ribera 2850, Concepción, Chile

E-mail address: jgarcias@ucsc.cl

C. Valls: Departamento de Matemática, Instituto Superior Técnico, Universidade de LisBoA, Av. Rovisco Pais, 1049-001, Lisboa, Portugal

E-mail address: cvalls@math.ist.utl.pt 